\title{
A hybrid iterative method for a combination of equilibria problem, a combination of variational inequality problems and a hierarchical fixed point problem
}

Abdellah Bnouhachem*

"Correspondence:

babedallah@yahoo.com

School of Management and

Engineering, Nanjing University,

Nanjing, 210093, P.R. China

ENSA, Ibn Zohr University, BP 1136,

Agadir, Morocco

\begin{abstract}
In this paper, we introduce and analyze a general iterative algorithm for finding a common solution of a combination of variational inequality problems, a combination of equilibria problem, and a hierarchical fixed point problem in the setting of real Hilbert space. Under appropriate conditions we derive the strong convergence results for this method. Several special cases are also discussed. Preliminary numerical experiments are included to verify the theoretical assertions of the proposed method. The results presented in this paper extend and improve some well-known results in the literature.
\end{abstract}

MSC: 49J30; 47H09; 47J20

Keywords: combination of equilibria problem; variational inequality; hierarchical fixed point problem; fixed point problem; projection method

\section{Introduction}

Let $H$ be a real Hilbert space, whose inner product and norm are denoted by $\langle\cdot, \cdot\rangle$ and $\|\cdot\|$. Let $C$ be a nonempty closed convex subset of $H$. Let $F_{1}: C \times C \rightarrow \mathbb{R}$ be a bifunction, the equilibrium problem is to find $x \in C$ such that

$$
F_{1}(x, y) \geq 0, \quad \forall y \in C,
$$

which was considered and investigated by Blum and Oettli [1]. The solution set of (1.1) is denoted by $\operatorname{EP}\left(F_{1}\right)$. Equilibrium problems theory provides us with a unified, natural, innovative, and general framework to study a wide class of problems arising in finance, economics, network analysis, transportation, elasticity, and optimization. This theory has witnessed an explosive growth in theoretical advances and applications across all disciplines of pure and applied sciences; see [2-11].

If $F_{1}(u, v)=\langle A u, v-u\rangle$, where $A: C \rightarrow H$ is a nonlinear operator, then problem (1.1) is equivalent to finding a vector $u \in C$ such that

$$
\langle v-u, A u\rangle \geq 0, \quad \forall v \in C,
$$

O2014 Bnouhachem; licensee Springer. This is an Open Access article distributed under the terms of the Creative Commons Attribution License (http://creativecommons.org/licenses/by/2.0), which permits unrestricted use, distribution, and reproduction in any medium, provided the original work is properly cited. 
which is known as the classical variational inequality. The solution of (1.2) is denoted by $\mathrm{VI}(C, A)$. It is easy to observe that

$$
u^{*} \in \mathrm{VI}(C, A) \quad \Longleftrightarrow \quad u^{*}=P_{C}\left[u^{*}-\rho A u^{*}\right], \quad \text { where } \rho>0
$$

Variational inequalities are being used as a mathematical programming tool in modeling a large class of problems arising in various branches of pure and applied sciences. In recent years, variational inequalities have been generalized and extended novel and new techniques in several directions. We now have a variety of techniques to suggest and analyze various iterative algorithms for solving variational inequalities and related optimization problems; see [1-33].

For $i=1,2, \ldots, N$, let $F_{i}: C \times C \rightarrow \mathbb{R}$ be bifunctions and $a_{i} \in(0,1)$ with $\sum_{i=1}^{N} a_{i}=1$. Define the mapping $\sum_{i=1}^{N} a_{i} F_{i}: C \times C \rightarrow \mathbb{R}$. The combination of equilibria problem is to find $x \in C$ such that

$$
\sum_{i=1}^{N} a_{i} F_{i}(x, y) \geq 0, \quad \forall y \in C,
$$

which was considered and investigated by Suwannaut and Kangtunyakarn [12]. The set of solutions (1.3) is denoted by

$$
\operatorname{EP}\left(\sum_{i=1}^{N} a_{i} F_{i}\right)=\bigcap_{i=1}^{N} \operatorname{EP}\left(F_{i}\right) .
$$

If $F_{i}=F_{1}, \forall i=1,2, \ldots, N$, then the combination of equilibria problem (1.3) reduces to the equilibrium problem (1.1).

For $i=1,2, \ldots, N$, let $A_{i}$ be a strongly positive linear bounded operator on a Hilbert space $H$ with coefficient $\rho_{i}>0$ and $b_{i} \in(0,1)$ with $\sum_{i=1}^{N} b_{i}=1$. The combination of variational inequality problems is to find $x \in C$ such that

$$
\left\langle\sum_{i=1}^{N} b_{i} A_{i} x, y-x\right\rangle \geq 0, \quad \forall y \in C .
$$

If $A_{i}=A, \forall i=1,2, \ldots, N$, then the combination of variational inequality problems (1.4) reduces to the variational inequality problem (1.2).

We introduce the following definitions, which are useful in the following analysis.

Definition 1.1 The mapping $T: C \rightarrow H$ is said to be

(a) monotone if

$$
\langle T x-T y, x-y\rangle \geq 0, \quad \forall x, y \in C
$$

(b) strongly monotone if there exists $\alpha>0$ such that

$$
\langle T x-T y, x-y\rangle \geq \alpha\|x-y\|^{2}, \quad \forall x, y \in C ;
$$


(c) strongly positive linear bounded if there exists $\alpha>0$ such that

$$
\langle T x, x\rangle \geq \alpha\|x\|^{2}, \quad \forall x \in C
$$

(d) nonexpansive if

$$
\|T x-T y\| \leq\|x-y\|, \quad \forall x, y \in C ;
$$

(e) $k$-Lipschitz continuous if there exists a constant $k>0$ such that

$$
\|T x-T y\| \leq k\|x-y\|, \quad \forall x, y \in C ;
$$

(f) a contraction on $C$ if there exists a constant $0 \leq k<1$ such that

$$
\|T x-T y\| \leq k\|x-y\|, \quad \forall x, y \in C .
$$

It is easy to observe that every $\alpha$-inverse-strongly monotone $T$ is monotone and Lipschitz continuous. It is well known that every nonexpansive operator $T: H \rightarrow H$ satisfies, for all $(x, y) \in H \times H$, the inequality

$$
\langle(x-T(x))-(y-T(y)), T(y)-T(x)\rangle \leq \frac{1}{2}\|(T(x)-x)-(T(y)-y)\|^{2}
$$

and therefore, we get, for all $(x, y) \in H \times \operatorname{Fix}(T)$,

$$
\langle x-T(x), y-T(x)\rangle \leq \frac{1}{2}\|T(x)-x\|^{2} .
$$

The fixed point problem for the mapping $T$ is to find $x \in C$ such that

$$
T x=x
$$

We denote by $F(T)$ the set of solutions of (1.7). It is well known that $F(T)$ is closed and convex, and $P_{F}(T)$ is well defined.

Let $S: C \rightarrow H$ be a nonexpansive mapping. The following problem is called a hierarchical fixed point problem: Find $x \in F(T)$ such that

$$
\langle x-S x, y-x\rangle \geq 0, \quad \forall y \in F(T) .
$$

It is well known that the hierarchical fixed point problem (1.8) links with some monotone variational inequalities and convex programming problems; see [13]. Various methods have been proposed to solve the hierarchical fixed point problem; see [14-21]. By combining Korpelevich's extragradient method and the viscosity approximation method, Ceng et al. [22] introduced and analyzed implicit and explicit iterative schemes for computing a common element of the set of fixed points of a nonexpansive mapping and the set of solutions of the variational inequality for an $\alpha$-inverse-strongly monotone mapping in a Hilbert space. Under suitable assumptions, they proved the strong convergence of the sequences generated by the proposed schemes. 
In 2010, Yao et al. [13] introduced the following strong convergence iterative algorithm to solve problem (1.8):

$$
\begin{aligned}
& y_{n}=\beta_{n} S x_{n}+\left(1-\beta_{n}\right) x_{n}, \\
& x_{n+1}=P_{C}\left[\alpha_{n} f\left(x_{n}\right)+\left(1-\alpha_{n}\right) T y_{n}\right], \quad \forall n \geq 0,
\end{aligned}
$$

where $f: C \rightarrow H$ is a contraction mapping and $\left\{\alpha_{n}\right\}$ and $\left\{\beta_{n}\right\}$ are two sequences in $(0,1)$. Under some certain restrictions on the parameters, Yao et al. proved that the sequence $\left\{x_{n}\right\}$ generated by (1.9) converges strongly to $z \in F(T)$, which is the unique solution of the following variational inequality:

$$
\langle(I-f) z, y-z\rangle \geq 0, \quad \forall y \in F(T) .
$$

In 2011, Ceng et al. [23] investigated the following iterative method:

$$
x_{n+1}=P_{C}\left[\alpha_{n} \rho U\left(x_{n}\right)+\left(I-\alpha_{n} \mu F\right)\left(T\left(y_{n}\right)\right)\right], \quad \forall n \geq 0,
$$

where $U$ is a Lipschitzian mapping, and $F$ is a Lipschitzian and strongly monotone mapping. They proved that under some approximate assumptions on the operators and parameters, the sequence $\left\{x_{n}\right\}$ generated by (1.11) converges strongly to the unique solution of the variational inequality

$$
\langle\rho U(z)-\mu F(z), x-z\rangle \geq 0, \quad \forall x \in \operatorname{Fix}(T) .
$$

Very recently, in 2013, Wang and Xu [24] investigated an iterative method for a hierarchical fixed point problem by

$$
\begin{aligned}
& y_{n}=\beta_{n} S x_{n}+\left(1-\beta_{n}\right) x_{n}, \\
& x_{n+1}=P_{C}\left[\alpha_{n} \rho U\left(x_{n}\right)+\left(I-\alpha_{n} \mu F\right)\left(T\left(y_{n}\right)\right)\right], \quad \forall n \geq 0,
\end{aligned}
$$

where $S: C \rightarrow C$ is a nonexpansive mapping. They proved that under some approximate assumptions on the operators and parameters, the sequence $\left\{x_{n}\right\}$ generated by (1.13) converges strongly to the unique solution of the variational inequality (1.12).

In this paper, motivated by the work of Ceng et al. [23, 26], Yao et al. [13], Wang and Xu [24], Bnouhachem $[15,25]$ and by the recent work going in this direction, we give an iterative method for finding the approximate element of the common set of solutions of (1.3), (1.4), and (1.8) in a real Hilbert space. We establish a strong convergence theorem based on this method. We would like to mention that our proposed method is quite general and flexible and includes many known results for solving of variational inequality problems, equilibrium problems, and hierarchical fixed point problems; see, e.g., [13, 16, 18, 23, 25, 27] and relevant references cited therein.

\section{Preliminaries}

In this section, we list some fundamental lemmas that are useful in the consequent analysis. The first lemma provides some basic properties of projection onto $C$. 
Lemma 2.1 Let $P_{C}$ denote the projection of $H$ onto $C$. Then we have the following inequalities:

$$
\begin{aligned}
& \left\langle z-P_{C}[z], P_{C}[z]-v\right\rangle \geq 0, \quad \forall z \in H, v \in C ; \\
& \left\langle u-v, P_{C}[u]-P_{C}[v]\right\rangle \geq\left\|P_{C}[u]-P_{C}[v]\right\|^{2}, \quad \forall u, v \in H ; \\
& \left\|P_{C}[u]-P_{C}[v]\right\| \leq\|u-v\|, \quad \forall u, v \in H ; \\
& \left\|u-P_{C}[z]\right\|^{2} \leq\|z-u\|^{2}-\left\|z-P_{C}[z]\right\|^{2}, \quad \forall z \in H, u \in C .
\end{aligned}
$$

Assumption 2.1 [1] Let $F_{1}: C \times C \rightarrow \mathbb{R}$ be a bifunction satisfying the following assumptions:

$\left(\mathrm{A}_{1}\right) F_{1}(x, x)=0, \forall x \in C$;

$\left(\mathrm{A}_{2}\right) F_{1}$ is monotone, i.e., $F_{1}(x, y)+F_{1}(y, x) \leq 0, \forall x, y \in C$;

$\left(\mathrm{A}_{3}\right)$ for each $x, y, z \in C, \lim _{t \rightarrow 0} F_{1}(t z+(1-t) x, y) \leq F_{1}(x, y)$;

$\left(\mathrm{A}_{4}\right)$ for each $x \in C, y \rightarrow F_{1}(x, y)$ is convex and lower semicontinuous.

Lemma 2.2 [2] Let $C$ be a nonempty closed convex subset of $H$. Let $F_{1}: C \times C \rightarrow \mathbb{R}$ satisfy $\left(\mathrm{A}_{1}\right)-\left(\mathrm{A}_{4}\right)$. Assume that for $r>0$ and $\forall x \in H$, define a mapping $T_{r}: H \rightarrow C$ as follows:

$$
T_{r}(x)=\left\{z \in C: F_{1}(z, y)+\frac{1}{r}\langle y-z, z-x\rangle \geq 0, \forall y \in C\right\} .
$$

Then the following hold:

(i) $T_{r}$ is nonempty and single-valued;

(ii) $T_{r}$ is firmly nonexpansive, i.e.;

$$
\left\|T_{r}(x)-T_{r}(y)\right\|^{2} \leq\left\langle T_{r}(x)-T_{r}(y), x-y\right\rangle, \quad \forall x, y \in H ;
$$

(iii) $F\left(T_{r}\right)=\mathrm{EP}\left(F_{1}\right)$;

(iv) $\mathrm{EP}\left(F_{1}\right)$ is closed and convex.

Lemma 2.3 [12] Let $C$ be a nonempty closed convex subset of a real Hilbert space H. For $i=1,2, \ldots, N$, let $F_{i}: C \times C \rightarrow \mathbb{R}$ be bifunctions satisfying $\left(\mathrm{A}_{1}\right)-\left(\mathrm{A}_{4}\right)$ with $\bigcap_{i=1}^{N} \mathrm{EP}\left(F_{i}\right) \neq \emptyset$. Then $\sum_{i=1}^{N} a_{i} F_{i}$ satisfies $\left(\mathrm{A}_{1}\right)-\left(\mathrm{A}_{4}\right)$ and

$$
\operatorname{Fix}\left(T_{r}\right)=\operatorname{EP}\left(\sum_{i=1}^{N} a_{i} F_{i}\right)=\bigcap_{i=1}^{N} \operatorname{EP}\left(F_{i}\right),
$$

where $a_{i} \in(0,1)$ for $i=1,2, \ldots, N$ and $\sum_{i=1}^{N} a_{i}=1$.

Lemma 2.4 [28] Let $C$ be a nonempty closed convex subset of a real Hilbert space $H$.

If $T: C \rightarrow C$ is a nonexpansive mapping with $\operatorname{Fix}(T) \neq \emptyset$, then the mapping $I-T$ is demiclosed at 0 , i.e., if $\left\{x_{n}\right\}$ is a sequence in $C$ weakly converging to $x$, and if $\left\{(I-T) x_{n}\right\}$ converges strongly to 0 , then $(I-T) x=0$. 
Lemma 2.5 [23] Let $U: C \rightarrow H$ be a $\tau$-Lipschitzian mapping, and let $F: C \rightarrow H$ be a $k$-Lipschitzian and $\eta$-strongly monotone mapping, then for $0 \leq \rho \tau<\mu \eta, \mu F-\rho U$ is $\mu \eta-$ $\rho \tau$-strongly monotone, i.e.,

$$
\langle(\mu F-\rho U) x-(\mu F-\rho U) y, x-y| \geq(\mu \eta-\rho \tau)\|x-y\|^{2}, \quad \forall x, y \in C .
$$

Lemma 2.6 [29] Suppose that $\lambda \in(0,1)$ and $\mu>0$. Let $F: C \rightarrow H$ be a $k$-Lipschitzian and $\eta$-strongly monotone operator. In association with a nonexpansive mapping $T: C \rightarrow C$, define the mapping $T^{\lambda}: C \rightarrow H$ by

$$
T^{\lambda} x=T x-\lambda \mu F T(x), \quad \forall x \in C .
$$

Then $T^{\lambda}$ is a contraction provided $\mu<\frac{2 \eta}{k^{2}}$, that is,

$$
\left\|T^{\lambda} x-T^{\lambda} y\right\| \leq(1-\lambda \nu)\|x-y\|, \quad \forall x, y \in C,
$$

where $v=1-\sqrt{1-\mu\left(2 \eta-\mu k^{2}\right)}$.

Lemma 2.7 [30] Assume that $\left\{a_{n}\right\}$ is a sequence of nonnegative real numbers such that

$$
a_{n+1} \leq\left(1-v_{n}\right) a_{n}+\delta_{n}
$$

where $\left\{v_{n}\right\}$ is a sequence in $(0,1)$ and $\delta_{n}$ is a sequence such that

(1) $\sum_{n=1}^{\infty} v_{n}=\infty$;

(2) $\lim \sup _{n \rightarrow \infty} \delta_{n} / v_{n} \leq 0$ or $\sum_{n=1}^{\infty}\left|\delta_{n}\right|<\infty$.

Then $\lim _{n \rightarrow \infty} a_{n}=0$.

Lemma 2.8 [31] Let C be a closed convex subset of $H$. Let $\left\{x_{n}\right\}$ be a bounded sequence in $H$. Assume that

(i) the weak $w$-limit set $w_{w}\left(x_{n}\right) \subset C$ where $w_{w}\left(x_{n}\right)=\left\{x: x_{n_{i}} \rightarrow x\right\}$;

(ii) for each $z \in C, \lim _{n \rightarrow \infty}\left\|x_{n}-z\right\|$ exists.

Then $\left\{x_{n}\right\}$ is weakly convergent to a point in $C$.

Lemma 2.9 [12] Let $C$ be a nonempty closed convex subset of a real Hilbert space H. For every $i=1,2, \ldots, N$, let $A_{i}$ be a strongly positive linear bounded operator on a Hilbert space $H$ with coefficient $\rho_{i}>0$, i.e., $\left\langle A_{i} x, x\right\rangle \geq \rho_{i}\|x\|^{2}, \forall x \in H$, and $\bar{\rho}=\min _{i=1,2, \ldots, N} \rho_{i}$. Let $\left\{b_{i}\right\}_{i=1}^{N} \subseteq$ $(0,1)$ with $\sum_{i=1}^{N} b_{i}=1$. Then the following properties hold:

(i) $\left\|I-\lambda \sum_{i=1}^{N} b_{i} A_{i}\right\| \leq 1-\lambda \bar{\rho}$ and $\left\|I-\lambda \sum_{i=1}^{N} b_{i} A_{i}\right\|$ is a nonexpansive mapping for every $0<\lambda<\left\|A_{i}\right\|^{-1}(i=1,2, \ldots, N)$.

(ii) $\operatorname{VI}\left(C, \sum_{i=1}^{N} b_{i} A_{i}\right)=\bigcap_{i=1}^{N} \operatorname{VI}\left(C, A_{i}\right)$.

\section{The proposed method and some properties}

In this section, we suggest and analyze our method for finding common solutions of the combination of equilibria problem (1.3), the combination of variational inequality problems (1.4), and the hierarchical fixed point problem (1.8).

Let $C$ be a nonempty closed convex subset of a real Hilbert space $H$. For $i=1,2, \ldots, N$, let $F_{i}: C \times C \rightarrow \mathbb{R}$ be bifunctions satisfying $\left(\mathrm{A}_{1}\right)-\left(\mathrm{A}_{4}\right)$, let $A_{i}$ be a strongly positive linear 
bounded operator on a Hilbert space $H$ with coefficient $\rho_{i}>0$ and $\bar{\rho}=\min _{i=1,2, \ldots, N} \rho_{i}$, and let $S, T: C \rightarrow C$ be nonexpansive mappings such that $F(T) \cap \bigcap_{i=1}^{N} \operatorname{EP}\left(F_{i}\right) \cap \bigcap_{i=1}^{N} \operatorname{VI}(C$, $\left.A_{i}\right) \neq \emptyset$. Let $F: C \rightarrow C$ be a $k$-Lipschitzian mapping and be $\eta$-strongly monotone, and let $U: C \rightarrow C$ be a $\tau$-Lipschitzian mapping.

Algorithm 3.1 For an arbitrarily given $x_{0} \in C$, let the iterative sequences $\left\{u_{n}\right\},\left\{x_{n}\right\},\left\{y_{n}\right\}$, and $\left\{z_{n}\right\}$ be generated by

$$
\left\{\begin{array}{l}
\sum_{i=1}^{N} a_{i} F_{i}\left(u_{n}, y\right)+\frac{1}{r_{n}}\left\langle y-u_{n}, u_{n}-x_{n}\right\rangle \geq 0, \quad \forall y \in C ; \\
z_{n}=P_{C}\left[u_{n}-\lambda_{n} \sum_{i=1}^{N} b_{i} A_{i} u_{n}\right] ; \\
y_{n}=\beta_{n} S x_{n}+\left(1-\beta_{n}\right) u_{n} ; \\
x_{n+1}=\gamma_{n} x_{n}+\left(1-\gamma_{n}\right) P_{C}\left[\alpha_{n} \rho U\left(x_{n}\right)+\left(I-\alpha_{n} \mu F\right)\left(T\left(y_{n}\right)\right)\right], \quad \forall n \geq 0 .
\end{array}\right.
$$

Suppose that the parameters satisfy $0<\mu<\frac{2 \eta}{k^{2}}, 0 \leq \rho \tau<\nu$, where $\nu=1-\sqrt{1-\mu\left(2 \eta-\mu k^{2}\right)}$. Also $\left\{\gamma_{n}\right\},\left\{\alpha_{n}\right\},\left\{\beta_{n}\right\}$, and $\left\{r_{n}\right\}$ are sequences in $(0,1)$ satisfying the following conditions:

(a) $0<a \leq \gamma_{n} \leq b<1$,

(b) $\lim _{n \rightarrow \infty} \alpha_{n}=0$ and $\sum_{n=1}^{\infty} \alpha_{n}=\infty$,

(c) $\lim _{n \rightarrow \infty}\left(\beta_{n} / \alpha_{n}\right)=0$,

(d) $\sum_{i=1}^{N} a_{i}=\sum_{i=1}^{N} b_{i}=1$,

(e) $\sum_{n=1}^{\infty}\left|\alpha_{n-1}-\alpha_{n}\right|<\infty, \sum_{n=1}^{\infty}\left|\gamma_{n-1}-\gamma_{n}\right|<\infty$, and $\sum_{n=1}^{\infty}\left|\beta_{n-1}-\beta_{n}\right|<\infty$,

(f) $\liminf _{n \rightarrow \infty} r_{n}>0$ and $\sum_{n=1}^{\infty}\left|r_{n-1}-r_{n}\right|<\infty$,

(g) $\lim _{n \rightarrow \infty} \lambda_{n}=0$ and $\sum_{n=1}^{\infty}\left|\lambda_{n-1}-\lambda_{n}\right|<\infty$.

If for $i=1,2, \ldots, N, F_{i}=F^{\prime}$ and $A_{i}=A$, then Algorithm 3.1 reduces to Algorithm 3.2 for finding the common solutions of equilibrium problem (1.1), variational inequality problem (1.2) and the hierarchical fixed point problem (1.8).

Algorithm 3.2 For an arbitrarily given $x_{0} \in C$ arbitrarily, let the iterative sequences $\left\{u_{n}\right\}$, $\left\{x_{n}\right\},\left\{y_{n}\right\}$, and $\left\{z_{n}\right\}$ be generated by

$$
\left\{\begin{array}{l}
F^{\prime}\left(u_{n}, y\right)+\frac{1}{r_{n}}\left\langle y-u_{n}, u_{n}-x_{n}\right\rangle \geq 0, \quad \forall y \in C ; \\
z_{n}=P_{C}\left[u_{n}-\lambda_{n} A u_{n}\right] ; \\
y_{n}=\beta_{n} S x_{n}+\left(1-\beta_{n}\right) z_{n} ; \\
x_{n+1}=\gamma_{n} x_{n}+\left(1-\gamma_{n}\right) P_{C}\left[\alpha_{n} \rho U\left(x_{n}\right)+\left(I-\alpha_{n} \mu F\right)\left(T\left(y_{n}\right)\right)\right], \quad \forall n \geq 0 .
\end{array}\right.
$$

Suppose that the parameters satisfy $0<\mu<\frac{2 \eta}{k^{2}}, 0 \leq \rho \tau<\nu$, where $\nu=1-\sqrt{1-\mu\left(2 \eta-\mu k^{2}\right)}$. Also $\left\{\gamma_{n}\right\},\left\{\alpha_{n}\right\},\left\{\beta_{n}\right\}$, and $\left\{r_{n}\right\}$ are sequences in $(0,1)$ satisfying the following conditions:

(a) $0<a \leq \gamma_{n} \leq b<1$,

(b) $\lim _{n \rightarrow \infty} \alpha_{n}=0$ and $\sum_{n=1}^{\infty} \alpha_{n}=\infty$,

(c) $\lim _{n \rightarrow \infty}\left(\beta_{n} / \alpha_{n}\right)=0$,

(d) $\sum_{n=1}^{\infty}\left|\alpha_{n-1}-\alpha_{n}\right|<\infty, \sum_{n=1}^{\infty}\left|\gamma_{n-1}-\gamma_{n}\right|<\infty$, and $\sum_{n=1}^{\infty}\left|\beta_{n-1}-\beta_{n}\right|<\infty$,

(e) $\liminf _{n \rightarrow \infty} r_{n}>0$ and $\sum_{n=1}^{\infty}\left|r_{n-1}-r_{n}\right|<\infty$,

(f) $\lim _{n \rightarrow \infty} \lambda_{n}=0$ and $\sum_{n=1}^{\infty}\left|\lambda_{n-1}-\lambda_{n}\right|<\infty$.

Remark 3.1 Our method can be viewed as an extension and improvement for some wellknown results, for example the following. 
- If $\gamma_{n}=0$, the proposed method is an extension and improvement of the method of Wang and $\mathrm{Xu}$ [24] and Bnouhachem [25] for finding the approximate element of the common set of solutions of a combination of variational inequality problems, a combination of equilibria problem and a hierarchical fixed point problem in a real Hilbert space.

- If we have the Lipschitzian mapping $U=f, F=I, \rho=\mu=1$, and $\gamma_{n}=0$, we obtain an extension and improvement of the method of Yao et al. [13] for finding the approximate element of the common set of solutions of a combination of variational inequality problems, a combination of equilibria problem and a hierarchical fixed point problem in a real Hilbert space.

- The contractive mapping $f$ with a coefficient $\alpha \in[0,1)$ in other papers [13, 27, 29] is extended to the cases of the Lipschitzian mapping $U$ with a coefficient constant $\gamma \in[0, \infty)$.

This shows that Algorithm 3.1 is quite general and unifying.

Lemma 3.1 Let $x^{*} \in F(T) \cap \bigcap_{i=1}^{N} \operatorname{EP}\left(F_{i}\right) \cap \bigcap_{i=1}^{N} \operatorname{VI}\left(C, A_{i}\right)$. Then $\left\{x_{n}\right\},\left\{u_{n}\right\},\left\{z_{n}\right\}$, and $\left\{y_{n}\right\}$ are bounded.

Proof Let $x^{*} \in F(T) \cap \bigcap_{i=1}^{N} \mathrm{EP}\left(F_{i}\right) \cap \bigcap_{i=1}^{N} \mathrm{VI}\left(C, A_{i}\right)$; we have $x^{*}=T_{r_{n}}\left(x^{*}\right)$. It follows from Lemmas 2.2 and 2.3 that $u_{n}=T_{r_{n}}\left(x_{n}\right)$. Since $T_{r_{n}}$ is nonexpansive mapping, we have

$$
\left\|u_{n}-x^{*}\right\| \leq\left\|x_{n}-x^{*}\right\|
$$

Since $\lim _{n \rightarrow \infty} \lambda_{n}=0$, without loss of generality, we may assume that $0<\lambda_{n}<\left\|A_{i}\right\|^{-1}, \forall n \geq$ 0 and $i=1,2, \ldots, N$, by Lemma 2.9 , the mapping $I-\lambda_{n} \sum_{i=1}^{N} b_{i} A_{i}$ is nonexpansive mapping, and we have

$$
\begin{aligned}
\left\|z_{n}-x^{*}\right\| & =\left\|P_{C}\left[u_{n}-\lambda_{n} \sum_{i=1}^{N} b_{i} A_{i} u_{n}\right]-P_{C}\left[x^{*}-\lambda_{n} \sum_{i=1}^{N} b_{i} A_{i} x^{*}\right]\right\| \\
& \leq\left\|\left(I-\lambda_{n} \sum_{i=1}^{N} b_{i} A_{i}\right) u_{n}-\left(I-\lambda_{n} \sum_{i=1}^{N} b_{i} A_{i}\right) x^{*}\right\| \\
& \leq\left\|u_{n}-x^{*}\right\| \\
& \leq\left\|x_{n}-x^{*}\right\| .
\end{aligned}
$$

We define $V_{n}=\alpha_{n} \rho U\left(x_{n}\right)+\left(I-\alpha_{n} \mu F\right)\left(T\left(y_{n}\right)\right)$. Next, we prove that the sequence $\left\{x_{n}\right\}$ is bounded, and without loss of generality we can assume that $\beta_{n} \leq \alpha_{n}$ for all $n \geq 1$. From (3.1), we have

$$
\begin{aligned}
\left\|x_{n+1}-x^{*}\right\|= & \left\|\gamma_{n}\left(x_{n}-x^{*}\right)+\left(1-\gamma_{n}\right)\left(P_{C}\left[V_{n}\right]-P_{C}\left[x^{*}\right]\right)\right\| \\
\leq & \gamma_{n}\left\|x_{n}-x^{*}\right\|+\left(1-\gamma_{n}\right)\left\|\alpha_{n} \rho U\left(x_{n}\right)+\left(I-\alpha_{n} \mu F\right)\left(T\left(y_{n}\right)\right)-x^{*}\right\| \\
\leq & \gamma_{n}\left\|x_{n}-x^{*}\right\|+\left(1-\gamma_{n}\right)\left(\alpha_{n}\left\|\rho U\left(x_{n}\right)-\mu F\left(x^{*}\right)\right\|\right. \\
& \left.\quad+\left\|\left(I-\alpha_{n} \mu F\right)\left(T\left(y_{n}\right)\right)-\left(I-\alpha_{n} \mu F\right) T\left(x^{*}\right)\right\|\right) \\
= & \gamma_{n}\left\|x_{n}-x^{*}\right\|+\left(1-\gamma_{n}\right)\left(\alpha_{n}\left\|\rho U\left(x_{n}\right)-\rho U\left(x^{*}\right)+(\rho U-\mu F) x^{*}\right\|\right.
\end{aligned}
$$




$$
\begin{aligned}
& \left.+\left\|\left(I-\alpha_{n} \mu F\right)\left(T\left(y_{n}\right)\right)-\left(I-\alpha_{n} \mu F\right) T\left(x^{*}\right)\right\|\right) \\
& \leq \gamma_{n}\left\|x_{n}-x^{*}\right\|+\left(1-\gamma_{n}\right)\left(\alpha_{n} \rho \tau\left\|x_{n}-x^{*}\right\|\right. \\
& \left.+\alpha_{n}\left\|(\rho U-\mu F) x^{*}\right\|+\left(1-\alpha_{n} v\right)\left\|y_{n}-x^{*}\right\|\right) \\
& =\gamma_{n}\left\|x_{n}-x^{*}\right\|+\left(1-\gamma_{n}\right)\left(\alpha_{n} \rho \tau\left\|x_{n}-x^{*}\right\|+\alpha_{n}\left\|(\rho U-\mu F) x^{*}\right\|\right. \\
& \left.+\left(1-\alpha_{n} v\right)\left\|\beta_{n} S x_{n}+\left(1-\beta_{n}\right) z_{n}-x^{*}\right\|\right) \\
& \leq \gamma_{n}\left\|x_{n}-x^{*}\right\|+\alpha_{n} \rho \tau\left(1-\gamma_{n}\right)\left\|x_{n}-x^{*}\right\|+\alpha_{n}\left(1-\gamma_{n}\right)\left\|(\rho U-\mu F) x^{*}\right\| \\
& +\left(1-\alpha_{n} v\right)\left(1-\gamma_{n}\right)\left(\beta_{n}\left\|S x_{n}-S x^{*}\right\|+\beta_{n}\left\|S x^{*}-x^{*}\right\|+\left(1-\beta_{n}\right)\left\|z_{n}-x^{*}\right\|\right) \\
& \leq \gamma_{n}\left\|x_{n}-x^{*}\right\|+\alpha_{n} \rho \tau\left(1-\gamma_{n}\right)\left\|x_{n}-x^{*}\right\|+\alpha_{n}\left(1-\gamma_{n}\right)\left\|(\rho U-\mu F) x^{*}\right\| \\
& +\left(1-\alpha_{n} v\right)\left(1-\gamma_{n}\right)\left(\beta_{n}\left\|S x_{n}-S x^{*}\right\|+\beta_{n}\left\|S x^{*}-x^{*}\right\|+\left(1-\beta_{n}\right)\left\|x_{n}-x^{*}\right\|\right) \\
& \leq \gamma_{n}\left\|x_{n}-x^{*}\right\|+\alpha_{n} \rho \tau\left(1-\gamma_{n}\right)\left\|x_{n}-x^{*}\right\|+\alpha_{n}\left(1-\gamma_{n}\right)\left\|(\rho U-\mu F) x^{*}\right\| \\
& +\left(1-\alpha_{n} v\right)\left(1-\gamma_{n}\right)\left(\beta_{n}\left\|x_{n}-x^{*}\right\|+\beta_{n}\left\|S x^{*}-x^{*}\right\|+\left(1-\beta_{n}\right)\left\|x_{n}-x^{*}\right\|\right) \\
& =\left(1-\alpha_{n}(v-\rho \tau)\left(1-\gamma_{n}\right)\right)\left\|x_{n}-x^{*}\right\|+\alpha_{n}\left(1-\gamma_{n}\right)\left\|(\rho U-\mu F) x^{*}\right\| \\
& +\left(1-\alpha_{n} \nu\right)\left(1-\gamma_{n}\right) \beta_{n}\left\|S x^{*}-x^{*}\right\| \\
& \leq\left(1-\alpha_{n}(v-\rho \tau)\left(1-\gamma_{n}\right)\right)\left\|x_{n}-x^{*}\right\| \\
& +\alpha_{n}\left(1-\gamma_{n}\right)\left\|(\rho U-\mu F) x^{*}\right\|+\beta_{n}\left(1-\gamma_{n}\right)\left\|S x^{*}-x^{*}\right\| \\
& \leq\left(1-\alpha_{n}(v-\rho \tau)\left(1-\gamma_{n}\right)\right)\left\|x_{n}-x^{*}\right\| \\
& +\alpha_{n}\left(1-\gamma_{n}\right)\left(\left\|(\rho U-\mu F) x^{*}\right\|+\left\|S x^{*}-x^{*}\right\|\right) \\
& =\left(1-\alpha_{n}(v-\rho \tau)\left(1-\gamma_{n}\right)\right)\left\|x_{n}-x^{*}\right\| \\
& +\frac{\alpha_{n}\left(1-\gamma_{n}\right)(\nu-\rho \tau)}{v-\rho \tau}\left(\left\|(\rho U-\mu F) x^{*}\right\|+\left\|S x^{*}-x^{*}\right\|\right) \\
& \leq \max \left\{\left\|x_{n}-x^{*}\right\|, \frac{1}{v-\rho \tau}\left(\left\|(\rho U-\mu F) x^{*}\right\|+\left\|S x^{*}-x^{*}\right\|\right)\right\},
\end{aligned}
$$

where the third inequality follows from Lemma 2.6 and the fifth inequality follows from (3.3). By induction on $n$, we obtain $\left\|x_{n}-x^{*}\right\| \leq \max \left\{\left\|x_{0}-x^{*}\right\|, \frac{1}{v-\rho \tau}\left(\left\|(\rho U-\mu F) x^{*}\right\|+\| S x^{*}-\right.\right.$ $\left.\left.x^{*} \|\right)\right\}$, for $n \geq 0$ and $x_{0} \in C$. Hence, $\left\{x_{n}\right\}$ is bounded and consequently, we deduce that $\left\{u_{n}\right\}$, $\left\{z_{n}\right\},\left\{v_{n}\right\},\left\{y_{n}\right\},\left\{S\left(x_{n}\right)\right\},\left\{T\left(x_{n}\right)\right\},\left\{F\left(T\left(y_{n}\right)\right)\right\}$, and $\left\{U\left(x_{n}\right)\right\}$ are bounded.

Lemma 3.2 Let $x^{*} \in F(T) \cap \bigcap_{i=1}^{N} \operatorname{EP}\left(F_{i}\right) \cap \bigcap_{i=1}^{N} \mathrm{VI}\left(C, A_{i}\right)$ and $\left\{x_{n}\right\}$ be the sequence generated by Algorithm 3.1. Then we have:

(a) $\lim _{n \rightarrow \infty}\left\|x_{n+1}-x_{n}\right\|=0$.

(b) The weak $w$-limit set $w_{w}\left(x_{n}\right) \subset F(T)\left(w_{w}\left(x_{n}\right)=\left\{x: x_{n_{i}} \rightarrow x\right\}\right)$.

Proof From the nonexpansivity of the mapping $I-\lambda_{n} \sum_{i=1}^{N} b_{i} A_{i}$ and $P_{C}$, we have

$$
\begin{aligned}
\left\|z_{n}-z_{n-1}\right\| & \leq\left\|\left(u_{n}-\lambda_{n} \sum_{i=1}^{N} b_{i} A_{i} u_{n}\right)-\left(u_{n-1}-\lambda_{n-1} \sum_{i=1}^{N} b_{i} A_{i} u_{n-1}\right)\right\| \\
& =\|\left(u_{n}-\lambda_{n} \sum_{i=1}^{N} b_{i} A_{i} u_{n}\right)-\left(u_{n-1}-\lambda_{n} \sum_{i=1}^{N} b_{i} A_{i} u_{n-1}\right)
\end{aligned}
$$




$$
\begin{aligned}
& -\left(\lambda_{n}-\lambda_{n-1}\right) \sum_{i=1}^{N} b_{i} A_{i} u_{n-1} \| \\
\leq & \left\|\left(u_{n}-\lambda_{n} \sum_{i=1}^{N} b_{i} A_{i} u_{n}\right)-\left(u_{n-1}-\lambda_{n} \sum_{i=1}^{N} b_{i} A_{i} u_{n-1}\right)\right\| \\
& +\left|\lambda_{n}-\lambda_{n-1}\right|\left\|\sum_{i=1}^{N} b_{i} A_{i} u_{n-1}\right\| \\
\leq & \left\|u_{n}-u_{n-1}\right\|+\left|\lambda_{n}-\lambda_{n-1}\right|\left\|\sum_{i=1}^{N} b_{i} A_{i} u_{n-1}\right\| .
\end{aligned}
$$

Next, we estimate that

$$
\begin{aligned}
\left\|y_{n}-y_{n-1}\right\|= & \left\|\beta_{n} S x_{n}+\left(1-\beta_{n}\right) z_{n}-\left(\beta_{n-1} S x_{n-1}+\left(1-\beta_{n-1}\right) z_{n-1}\right)\right\| \\
= & \| \beta_{n}\left(S x_{n}-S x_{n-1}\right)+\left(\beta_{n}-\beta_{n-1}\right) S x_{n-1} \\
& +\left(1-\beta_{n}\right)\left(z_{n}-z_{n-1}\right)+\left(\beta_{n-1}-\beta_{n}\right) z_{n-1} \| \\
\leq & \beta_{n}\left\|x_{n}-x_{n-1}\right\|+\left(1-\beta_{n}\right)\left\|z_{n}-z_{n-1}\right\| \\
& +\left|\beta_{n}-\beta_{n-1}\right|\left(\left\|S x_{n-1}\right\|+\left\|z_{n-1}\right\|\right) .
\end{aligned}
$$

It follows from (3.4) and (3.5) that

$$
\begin{aligned}
\left\|y_{n}-y_{n-1}\right\| \leq & \beta_{n}\left\|x_{n}-x_{n-1}\right\|+\left(1-\beta_{n}\right)\left\{\left\|u_{n}-u_{n-1}\right\|+\left|\lambda_{n}-\lambda_{n-1}\right|\left\|\sum_{i=1}^{N} b_{i} A_{i} u_{n-1}\right\|\right\} \\
& +\left|\beta_{n}-\beta_{n-1}\right|\left(\left\|S x_{n-1}\right\|+\left\|z_{n-1}\right\|\right) .
\end{aligned}
$$

On the other hand, $u_{n}=T_{r_{n}}\left(x_{n}\right)$ and $u_{n-1}=T_{r_{n-1}}\left(x_{n-1}\right)$, we obtain

$$
\sum_{i=1}^{N} a_{i} F_{i}\left(u_{n}, y\right)+\frac{1}{r_{n}}\left\langle y-u_{n}, u_{n}-x_{n}\right\rangle \geq 0, \quad \forall y \in C
$$

and

$$
\sum_{i=1}^{N} a_{i} F_{i}\left(u_{n-1}, y\right)+\frac{1}{r_{n-1}}\left\langle y-u_{n-1}, u_{n-1}-x_{n-1}\right\rangle \geq 0, \quad \forall y \in C .
$$

Taking $y=u_{n-1}$ in (3.7) and $y=u_{n}$ in (3.8), we get

$$
\sum_{i=1}^{N} a_{i} F_{i}\left(u_{n}, u_{n-1}\right)+\frac{1}{r_{n}}\left\langle u_{n-1}-u_{n}, u_{n}-x_{n}\right\rangle \geq 0
$$

and

$$
\sum_{i=1}^{N} a_{i} F_{i}\left(u_{n-1}, u_{n}\right)+\frac{1}{r_{n-1}}\left\langle u_{n}-u_{n-1}, u_{n-1}-x_{n-1}\right\rangle \geq 0 .
$$


Adding (3.9) and (3.10) and using the monotonicity of $\sum_{i=1}^{N} a_{i} F_{i}$, we have

$$
\left\langle u_{n}-u_{n-1}, \frac{u_{n-1}-x_{n-1}}{r_{n-1}}-\frac{u_{n}-x_{n}}{r_{n}}\right\rangle \geq 0
$$

which implies that

$$
\begin{aligned}
0 & \leq\left\langle u_{n}-u_{n-1}, \frac{r_{n}}{r_{n-1}}\left(u_{n-1}-x_{n-1}\right)-\left(u_{n}-x_{n}\right)\right\rangle \\
& =\left\langle u_{n-1}-u_{n}, u_{n}-u_{n-1}+\left(1-\frac{r_{n}}{r_{n-1}}\right) u_{n-1}-x_{n}+\frac{r_{n}}{r_{n-1}} x_{n-1}\right\rangle \\
& =\left\langle u_{n-1}-u_{n},\left(1-\frac{r_{n}}{r_{n-1}}\right) u_{n-1}-x_{n}+\frac{r_{n}}{r_{n-1}} x_{n-1}\right\rangle-\left\|u_{n}-u_{n-1}\right\|^{2} \\
& =\left\langle u_{n-1}-u_{n},\left(1-\frac{r_{n}}{r_{n-1}}\right)\left(u_{n-1}-x_{n-1}\right)+\left(x_{n-1}-x_{n}\right)\right\rangle-\left\|u_{n}-u_{n-1}\right\|^{2} \\
& \leq\left\|u_{n-1}-u_{n}\right\|\left\{\left|1-\frac{r_{n}}{r_{n-1}}\right|\left\|u_{n-1}-x_{n-1}\right\|+\left\|x_{n-1}-x_{n}\right\|\right\}-\left\|u_{n}-u_{n-1}\right\|^{2}
\end{aligned}
$$

and then

$$
\left\|u_{n-1}-u_{n}\right\| \leq\left|1-\frac{r_{n}}{r_{n-1}}\right|\left\|u_{n-1}-x_{n-1}\right\|+\left\|x_{n-1}-x_{n}\right\| .
$$

Without loss of generality, let us assume that there exists a real number $\chi$ such that $r_{n}>$ $\chi>0$ for all positive integers $n$. Then we get

$$
\left\|u_{n-1}-u_{n}\right\| \leq\left\|x_{n-1}-x_{n}\right\|+\frac{1}{\chi}\left|r_{n-1}-r_{n}\right|\left\|u_{n-1}-x_{n-1}\right\| .
$$

It follows from (3.6) and (3.11) that

$$
\begin{aligned}
\left\|y_{n}-y_{n-1}\right\| \leq & \beta_{n}\left\|x_{n}-x_{n-1}\right\|+\left(1-\beta_{n}\right)\left\{\left\|x_{n}-x_{n-1}\right\|+\frac{1}{\chi}\left|r_{n}-r_{n-1}\right|\left\|u_{n-1}-x_{n-1}\right\|\right. \\
& \left.+\left|\lambda_{n}-\lambda_{n-1}\right|\left\|\sum_{i=1}^{N} b_{i} A_{i} u_{n-1}\right\|\right\}+\left|\beta_{n}-\beta_{n-1}\right|\left(\left\|S x_{n-1}\right\|+\left\|z_{n-1}\right\|\right) \\
= & \left\|x_{n}-x_{n-1}\right\|+\left(1-\beta_{n}\right)\left\{\frac{1}{\chi}\left|r_{n}-r_{n-1}\right|\left\|u_{n-1}-x_{n-1}\right\|\right. \\
& \left.+\left|\lambda_{n}-\lambda_{n-1}\right|\left\|\sum_{i=1}^{N} b_{i} A_{i} u_{n-1}\right\|\right\}+\left|\beta_{n}-\beta_{n-1}\right|\left(\left\|S x_{n-1}\right\|+\left\|z_{n-1}\right\|\right) .
\end{aligned}
$$

Next, we estimate that

$$
\begin{aligned}
\left\|x_{n+1}-x_{n}\right\|= & \left\|\left(\gamma_{n} x_{n}+\left(1-\gamma_{n}\right) P_{C}\left[V_{n}\right]\right)-\left(\gamma_{n-1} x_{n-1}+\left(1-\gamma_{n-1}\right) P_{C}\left[V_{n-1}\right]\right)\right\| \\
= & \| \gamma_{n}\left(x_{n}-x_{n-1}\right)+\left(\gamma_{n}-\gamma_{n-1}\right) x_{n-1}+\left(1-\gamma_{n}\right)\left(P_{C}\left[V_{n}\right]-P_{C}\left[V_{n-1}\right]\right) \\
& -\left(\gamma_{n}-\gamma_{n-1}\right) P_{C}\left[V_{n-1}\right] \|
\end{aligned}
$$




$$
\begin{aligned}
\leq & \gamma_{n}\left\|x_{n}-x_{n-1}\right\|+\left|\gamma_{n}-\gamma_{n-1}\right|\left(\left\|x_{n-1}\right\|+\left\|P_{C}\left[V_{n-1}\right]\right\|\right) \\
& +\left(1-\gamma_{n}\right)\left\|P_{C}\left[V_{n}\right]-P_{C}\left[V_{n-1}\right]\right\| .
\end{aligned}
$$

Applying Lemma 2.6 to get

$$
\begin{aligned}
\left\|P_{C}\left[V_{n}\right]-P_{C}\left[V_{n-1}\right]\right\| \leq & \| \alpha_{n} \rho\left(U\left(x_{n}\right)-U\left(x_{n-1}\right)\right)+\left(\alpha_{n}-\alpha_{n-1}\right) \rho U\left(x_{n-1}\right) \\
& +\left(I-\alpha_{n} \mu F\right)\left(T\left(y_{n}\right)\right)-\left(I-\alpha_{n} \mu F\right) T\left(y_{n-1}\right) \\
& +\left(I-\alpha_{n} \mu F\right)\left(T\left(y_{n-1}\right)\right)-\left(I-\alpha_{n-1} \mu F\right)\left(T\left(y_{n-1}\right)\right) \| \\
\leq & \alpha_{n} \rho \tau\left\|x_{n}-x_{n-1}\right\|+\left(1-\alpha_{n} \nu\right)\left\|y_{n}-y_{n-1}\right\| \\
& +\left|\alpha_{n}-\alpha_{n-1}\right|\left(\left\|\rho U\left(x_{n-1}\right)\right\|+\left\|\mu F\left(T\left(y_{n-1}\right)\right)\right\|\right) .
\end{aligned}
$$

From (3.12) and (3.14), we have

$$
\begin{aligned}
\left\|P_{C}\left[V_{n}\right]-P_{C}\left[V_{n-1}\right]\right\| \leq & \alpha_{n} \rho \tau\left\|x_{n}-x_{n-1}\right\| \\
& +\left(1-\alpha_{n} v\right)\left(\left\|x_{n}-x_{n-1}\right\|+\frac{1}{\mu}\left|r_{n}-r_{n-1}\right|\left\|u_{n-1}-x_{n-1}\right\|\right. \\
& \left.+\left|\lambda_{n}-\lambda_{n-1}\right|\left\|\sum_{i=1}^{N} b_{i} A_{i} u_{n-1}\right\|\right) \\
& +\left|\beta_{n}-\beta_{n-1}\right|\left(\left\|S x_{n-1}\right\|+\left\|z_{n-1}\right\|\right) \\
& +\left|\alpha_{n}-\alpha_{n-1}\right|\left(\left\|\rho U\left(x_{n-1}\right)\right\|+\left\|\mu F\left(T\left(y_{n-1}\right)\right)\right\|\right) \\
\leq & \left(1-(v-\rho \tau) \alpha_{n}\right)\left\|x_{n}-x_{n-1}\right\|+\frac{1}{\mu}\left|r_{n}-r_{n-1}\right|\left\|u_{n-1}-x_{n-1}\right\| \\
& +\left|\lambda_{n}-\lambda_{n-1}\right|\left\|\sum_{i=1}^{N} b_{i} A_{i} u_{n-1}\right\|+\left|\beta_{n}-\beta_{n-1}\right|\left(\left\|S x_{n-1}\right\|+\left\|z_{n-1}\right\|\right) \\
& +\left|\alpha_{n}-\alpha_{n-1}\right|\left(\left\|\rho U\left(x_{n-1}\right)\right\|+\left\|\mu F\left(T\left(y_{n-1}\right)\right)\right\|\right) .
\end{aligned}
$$

Substituting (3.15) into (3.13), we get

$$
\begin{aligned}
\left\|x_{n+1}-x_{n}\right\| \leq & \left(1-(v-\rho \tau)\left(1-\gamma_{n}\right) \alpha_{n}\right)\left\|x_{n}-x_{n-1}\right\|+\frac{1}{\mu}\left|r_{n}-r_{n-1}\right|\left\|u_{n-1}-x_{n-1}\right\| \\
& +\left|\lambda_{n}-\lambda_{n-1}\right|\left\|\sum_{i=1}^{N} b_{i} A_{i} u_{n-1}\right\|+\left|\beta_{n}-\beta_{n-1}\right|\left(\left\|S x_{n-1}\right\|+\left\|z_{n-1}\right\|\right) \\
& +\left|\alpha_{n}-\alpha_{n-1}\right|\left(\left\|\rho U\left(x_{n-1}\right)\right\|+\left\|\mu F\left(T\left(y_{n-1}\right)\right)\right\|\right) \\
& +\left|\gamma_{n}-\gamma_{n-1}\right|\left(\left\|x_{n-1}\right\|+\left\|P_{C}\left[V_{n-1}\right]\right\|\right) \\
\leq & \left(1-(v-\rho \tau)\left(1-\gamma_{n}\right) \alpha_{n}\right)\left\|x_{n}-x_{n-1}\right\| \\
& +M\left(\frac{1}{\mu}\left|r_{n}-r_{n-1}\right|+\left|\lambda_{n}-\lambda_{n-1}\right|+\left|\beta_{n}-\beta_{n-1}\right|\right. \\
& \left.+\left|\alpha_{n}-\alpha_{n-1}\right|+\left|\gamma_{n}-\gamma_{n-1}\right|\right) .
\end{aligned}
$$


Here

$$
\begin{aligned}
M= & \max \left\{\sup _{n \geq 1}\left\|u_{n-1}-x_{n-1}\right\|, \sup _{n \geq 1}\left\|\sum_{i=1}^{N} b_{i} A_{i} u_{n-1}\right\|, \sup _{n \geq 1}\left(\left\|S x_{n-1}\right\|+\left\|z_{n-1}\right\|\right),\right. \\
& \left.\sup _{n \geq 1}\left(\left\|\rho U\left(x_{n-1}\right)\right\|+\left\|\mu F\left(T\left(y_{n-1}\right)\right)\right\|\right), \sup _{n \geq 1}\left(\left\|x_{n-1}\right\|+\left\|P_{C}\left[V_{n-1}\right]\right\|\right)\right\} .
\end{aligned}
$$

It follows by conditions (a)-(b), (e)-(g) of Algorithm 3.1 and Lemma 2.7 that

$$
\lim _{n \rightarrow \infty}\left\|x_{n+1}-x_{n}\right\|=0
$$

Since $x_{n+1}-x_{n}=\left(1-\gamma_{n}\right)\left(P_{C}\left[V_{n}\right]-x_{n}\right)$, we obtain

$$
\lim _{n \rightarrow \infty}\left\|P_{C}\left[V_{n}\right]-x_{n}\right\|=0
$$

Next, we show that $\lim _{n \rightarrow \infty}\left\|u_{n}-x_{n}\right\|=0$. Since $T_{r_{n}}$ is firmly nonexpansive, we have

$$
\begin{aligned}
\left\|u_{n}-x^{*}\right\|^{2} & =\left\|T_{r_{n}}\left(x_{n}\right)-T_{r_{n}}\left(x^{*}\right)\right\|^{2} \\
& \leq\left\langle u_{n}-x^{*}, x_{n}-x^{*}\right\rangle \\
& =\frac{1}{2}\left\{\left\|u_{n}-x^{*}\right\|^{2}+\left\|x_{n}-x^{*}\right\|^{2}-\left\|u_{n}-x^{*}-\left(x_{n}-x^{*}\right)\right\|^{2}\right\} .
\end{aligned}
$$

Hence, we get

$$
\left\|u_{n}-x^{*}\right\|^{2} \leq\left\|x_{n}-x^{*}\right\|^{2}-\left\|u_{n}-x_{n}\right\|^{2}
$$

From (3.3) and the inequality above, we have

$$
\begin{aligned}
\left\|P_{C}\left[V_{n}\right]-x^{*}\right\|^{2}= & \left\langle P_{C}\left[V_{n}\right]-x^{*}, P_{C}\left[V_{n}\right]-x^{*}\right\rangle \\
= & \left\langle P_{C}\left[V_{n}\right]-V_{n}, P_{C}\left[V_{n}\right]-x^{*}\right\rangle+\left\langle V_{n}-x^{*}, P_{C}\left[V_{n}\right]-x^{*}\right\rangle \\
\leq & \left\langle\alpha_{n}\left(\rho U\left(x_{n}\right)-\mu F\left(x^{*}\right)\right)+\left(I-\alpha_{n} \mu F\right)\left(T\left(y_{n}\right)\right)\right. \\
& \left.-\left(I-\alpha_{n} \mu F\right)\left(T\left(x^{*}\right)\right), P_{C}\left[V_{n}\right]-x^{*}\right\rangle \\
= & \left\langle\alpha_{n} \rho\left(U\left(x_{n}\right)-U\left(x^{*}\right)\right), P_{C}\left[V_{n}\right]-x^{*}\right\rangle \\
& +\alpha_{n}\left\langle\rho U\left(x^{*}\right)-\mu F\left(x^{*}\right), P_{C}\left[V_{n}\right]-x^{*}\right\rangle \\
& +\left\langle\left(I-\alpha_{n} \mu F\right)\left(T\left(y_{n}\right)\right)-\left(I-\alpha_{n} \mu F\right)\left(T\left(x^{*}\right)\right), P_{C}\left[V_{n}\right]-x^{*}\right\rangle \\
\leq & \alpha_{n} \rho \tau\left\|x_{n}-x^{*}\right\|\left\|P_{C}\left[V_{n}\right]-x^{*}\right\|+\alpha_{n}\left\langle\rho U\left(x^{*}\right)-\mu F\left(x^{*}\right), P_{C}\left[V_{n}\right]-x^{*}\right\rangle \\
& +\left(1-\alpha_{n} \nu\right)\left\|y_{n}-x^{*}\right\|\left\|P_{C}\left[V_{n}\right]-x^{*}\right\| \\
\leq & \frac{\alpha_{n} \rho \tau}{2}\left(\left\|x_{n}-x^{*}\right\|^{2}+\left\|P_{C}\left[V_{n}\right]-x^{*}\right\|^{2}\right) \\
& +\alpha_{n}\left\langle\rho U\left(x^{*}\right)-\mu F\left(x^{*}\right), P_{C}\left[V_{n}\right]-x^{*}\right\rangle \\
& +\frac{\left(1-\alpha_{n} \nu\right)}{2}\left(\left\|y_{n}-x^{*}\right\|^{2}+\left\|P_{C}\left[V_{n}\right]-x^{*}\right\|^{2}\right)
\end{aligned}
$$




$$
\begin{aligned}
\leq & \frac{\left(1-\alpha_{n}(v-\rho \tau)\right)}{2}\left\|P_{C}\left[V_{n}\right]-x^{*}\right\|^{2}+\frac{\alpha_{n} \rho \tau}{2}\left\|x_{n}-x^{*}\right\|^{2} \\
& +\alpha_{n}\left\langle\rho U\left(x^{*}\right)-\mu F\left(x^{*}\right), P_{C}\left[V_{n}\right]-x^{*}\right\rangle \\
& +\frac{\left(1-\alpha_{n} v\right)}{2}\left(\beta_{n}\left\|S x_{n}-x^{*}\right\|^{2}+\left(1-\beta_{n}\right)\left\|z_{n}-x^{*}\right\|^{2}\right) \\
\leq & \frac{\left(1-\alpha_{n}(v-\rho \tau)\right)}{2}\left\|P_{C}\left[V_{n}\right]-x^{*}\right\|^{2}+\frac{\alpha_{n} \rho \tau}{2}\left\|x_{n}-x^{*}\right\|^{2} \\
& +\alpha_{n}\left\langle\rho U\left(x^{*}\right)-\mu F\left(x^{*}\right), P_{C}\left[V_{n}\right]-x^{*}\right\rangle+\frac{\left(1-\alpha_{n} v\right) \beta_{n}}{2}\left\|S x_{n}-x^{*}\right\|^{2} \\
& +\frac{\left(1-\alpha_{n} v\right)\left(1-\beta_{n}\right)}{2}\left\{\left\|x_{n}-x^{*}\right\|^{2}-\left\|u_{n}-x_{n}\right\|^{2}\right\},
\end{aligned}
$$

which implies that

$$
\begin{aligned}
\left\|P_{C}\left[V_{n}\right]-x^{*}\right\|^{2} \leq & \frac{\alpha_{n} \rho \tau}{1+\alpha_{n}(v-\rho \tau)}\left\|x_{n}-x^{*}\right\|^{2} \\
& +\frac{2 \alpha_{n}}{1+\alpha_{n}(v-\rho \tau)}\left\{\rho U\left(x^{*}\right)-\mu F\left(x^{*}\right), P_{C}\left[V_{n}\right]-x^{*}\right\rangle \\
& +\frac{\left(1-\alpha_{n} v\right) \beta_{n}}{1+\alpha_{n}(v-\rho \tau)}\left\|S x_{n}-x^{*}\right\|^{2} \\
& +\frac{\left(1-\alpha_{n} v\right)\left(1-\beta_{n}\right)}{1+\alpha_{n}(v-\rho \tau)}\left\{\left\|x_{n}-x^{*}\right\|^{2}-\left\|u_{n}-x_{n}\right\|^{2}\right\} \\
\leq & \frac{\alpha_{n} \rho \tau}{1+\alpha_{n}(v-\rho \tau)}\left\|x_{n}-x^{*}\right\|^{2} \\
& +\frac{2 \alpha_{n}}{1+\alpha_{n}(v-\rho \tau)}\left\langle\rho U\left(x^{*}\right)-\mu F\left(x^{*}\right), P_{C}\left[V_{n}\right]-x^{*}\right\rangle \\
& +\frac{\left(1-\alpha_{n} v\right) \beta_{n}}{1+\alpha_{n}(v-\rho \tau)}\left\|S x_{n}-x^{*}\right\|^{2} \\
& +\left\|x_{n}-x^{*}\right\|^{2}-\frac{\left(1-\alpha_{n} v\right)\left(1-\beta_{n}\right)}{1+\alpha_{n}(v-\rho \tau)}\left\|u_{n}-x_{n}\right\|^{2} .
\end{aligned}
$$

Hence,

$$
\begin{aligned}
& \frac{\left(1-\alpha_{n} v\right)\left(1-\beta_{n}\right)}{1+\alpha_{n}(v-\rho \tau)}\left\|u_{n}-x_{n}\right\|^{2} \\
& \leq \frac{\alpha_{n} \rho \tau}{1+\alpha_{n}(v-\rho \tau)}\left\|x_{n}-x^{*}\right\|^{2} \\
& \quad+\frac{2 \alpha_{n}}{1+\alpha_{n}(v-\rho \tau)}\left\langle\rho U\left(x^{*}\right)-\mu F\left(x^{*}\right), P_{C}\left[V_{n}\right]-x^{*}\right\rangle \\
& \quad+\frac{\left(1-\alpha_{n} v\right) \beta_{n}}{1+\alpha_{n}(v-\rho \tau)}\left\|S x_{n}-x^{*}\right\|^{2}+\left\|x_{n}-x^{*}\right\|^{2}-\left\|P_{C}\left[V_{n}\right]-x^{*}\right\|^{2} \\
& \leq \\
& \quad \frac{\alpha_{n} \rho \tau}{1+\alpha_{n}(v-\rho \tau)}\left\|x_{n}-x^{*}\right\|^{2}+\frac{\left(1-\alpha_{n} v\right) \beta_{n}}{1+\alpha_{n}(v-\rho \tau)}\left\|S x_{n}-x^{*}\right\|^{2} \\
& \quad+\frac{2 \alpha_{n}}{1+\alpha_{n}(v-\rho \tau)}\left\langle\rho U\left(x^{*}\right)-\mu F\left(x^{*}\right), P_{C}\left[V_{n}\right]-x^{*}\right\rangle \\
& \quad+\left(\left\|x_{n}-x^{*}\right\|+\left\|P_{C}\left[V_{n}\right]-x^{*}\right\|\right)\left\|P_{C}\left[V_{n}\right]-x_{n}\right\| .
\end{aligned}
$$


Since $\lim _{n \rightarrow \infty}\left\|P_{C}\left[V_{n}\right]-x_{n}\right\|=0, \alpha_{n} \rightarrow 0, \beta_{n} \rightarrow 0$, we obtain

$$
\lim _{n \rightarrow \infty}\left\|u_{n}-x_{n}\right\|=0
$$

By (2.2) and the nonexpansivity of the mapping $I-\lambda_{n} \sum_{i=1}^{N} b_{i} A_{i}$, we get

$$
\begin{aligned}
\left\|z_{n}-x^{*}\right\|^{2}= & \left\|P_{C}\left[u_{n}-\lambda_{n} \sum_{i=1}^{N} b_{i} A_{i} u_{n}\right]-P_{C}\left[x^{*}-\lambda_{n} \sum_{i=1}^{N} b_{i} A_{i} x^{*}\right]\right\|^{2} \\
\leq & \left\langle z_{n}-x^{*},\left(u_{n}-\lambda_{n} \sum_{i=1}^{N} b_{i} A_{i} u_{n}\right)-\left(x^{*}-\lambda_{n} \sum_{i=1}^{N} b_{i} A_{i} x^{*}\right)\right\rangle \\
= & \frac{1}{2}\left\{\left\|z_{n}-x^{*}\right\|^{2}+\left\|\left(I-\lambda_{n} \sum_{i=1}^{N} b_{i} A_{i}\right) u_{n}-\left(I-\lambda_{n} \sum_{i=1}^{N} b_{i} A_{i}\right) x^{*}\right\|^{2}\right. \\
& \left.-\left\|u_{n}-x^{*}-\lambda_{n}\left(\sum_{i=1}^{N} b_{i} A_{i} u_{n}-\sum_{i=1}^{N} b_{i} A_{i} x^{*}\right)-\left(z_{n}-x^{*}\right)\right\|^{2}\right\} \\
\leq & \frac{1}{2}\left\{\left\|z_{n}-x^{*}\right\|^{2}+\left\|u_{n}-x^{*}\right\|^{2}\right. \\
& \left.+\left\|u_{n}-z_{n}-\lambda_{n}\left(\sum_{i=1}^{N} b_{i} A_{i} u_{n}-\sum_{i=1}^{N} b_{i} A_{i} x^{*}\right)\right\|^{2}\right\} \\
& \left.+2 \lambda_{n}\left\langle u_{n}-z_{n}, \sum_{i=1}^{N} b_{i} A_{i} u_{n}-\sum_{i=1}^{N} b_{i} A_{i} x^{*}\right\rangle\right\} \\
\leq & \frac{1}{2}\left\{\left\|z_{n}-x_{n}^{*}\right\|^{2}+\left\|u_{n}-x^{*}\right\|^{2}+\left\|u_{n}-x^{*}\right\|^{2}-\left\|u_{n}-z_{n}\right\|^{2} z_{n} \|^{2}\right. \\
& \\
& \\
& \\
& \\
&
\end{aligned}
$$

Hence

$$
\begin{aligned}
\left\|z_{n}-x^{*}\right\|^{2} & \leq\left\|u_{n}-x^{*}\right\|^{2}-\left\|u_{n}-z_{n}\right\|^{2}+2 \lambda_{n}\left\|u_{n}-z_{n}\right\|\left\|\sum_{i=1}^{N} b_{i} A_{i} u_{n}-\sum_{i=1}^{N} b_{i} A_{i} x^{*}\right\| \\
& \leq\left\|x_{n}-x^{*}\right\|^{2}-\left\|u_{n}-z_{n}\right\|^{2}+2 \lambda_{n}\left\|u_{n}-z_{n}\right\|\left\|\sum_{i=1}^{N} b_{i} A_{i} u_{n}-\sum_{i=1}^{N} b_{i} A_{i} x^{*}\right\|,
\end{aligned}
$$

where the second inequality follows from (3.2). From (3.18), and the inequality above, we have

$$
\begin{aligned}
\left\|P_{C}\left[V_{n}\right]-x^{*}\right\|^{2} \leq & \frac{\left(1-\alpha_{n}(\nu-\rho \tau)\right)}{2}\left\|P_{C}\left[V_{n}\right]-x^{*}\right\|^{2}+\frac{\alpha_{n} \rho \tau}{2}\left\|x_{n}-x^{*}\right\|^{2} \\
& +\alpha_{n}\left\langle\rho U\left(x^{*}\right)-\mu F\left(x^{*}\right), P_{C}\left[V_{n}\right]-x^{*}\right\rangle
\end{aligned}
$$




$$
\begin{aligned}
& +\frac{\left(1-\alpha_{n} \nu\right)}{2}\left(\beta_{n}\left\|S x_{n}-x^{*}\right\|^{2}+\left(1-\beta_{n}\right)\left\|z_{n}-x^{*}\right\|^{2}\right) \\
\leq & \frac{\left(1-\alpha_{n}(v-\rho \tau)\right)}{2}\left\|P_{C}\left[V_{n}\right]-x^{*}\right\|^{2}+\frac{\alpha_{n} \rho \tau}{2}\left\|x_{n}-x^{*}\right\|^{2} \\
& +\alpha_{n}\left\langle\rho U\left(x^{*}\right)-\mu F\left(x^{*}\right), P_{C}\left[V_{n}\right]-x^{*}\right\rangle \\
& +\frac{\left(1-\alpha_{n} \nu\right)}{2}\left\{\beta_{n}\left\|S x_{n}-x^{*}\right\|^{2}+\left(1-\beta_{n}\right)\left(\left\|x_{n}-x^{*}\right\|^{2}\right.\right. \\
& \left.\left.-\left\|u_{n}-z_{n}\right\|^{2}+2 \lambda_{n}\left\|u_{n}-z_{n}\right\|\left\|\sum_{i=1}^{N} b_{i} A_{i} u_{n}-\sum_{i=1}^{N} b_{i} A_{i} x^{*}\right\|\right)\right\},
\end{aligned}
$$

which implies that

$$
\begin{aligned}
\left\|P_{C}\left[V_{n}\right]-x^{*}\right\|^{2} \leq & \frac{\alpha_{n} \rho \tau}{1+\alpha_{n}(\nu-\rho \tau)}\left\|x_{n}-x^{*}\right\|^{2} \\
& +\frac{2 \alpha_{n}}{1+\alpha_{n}(v-\rho \tau)}\left\langle\rho U\left(x^{*}\right)-\mu F\left(x^{*}\right), P_{C}\left[V_{n}\right]-x^{*}\right\rangle \\
& +\frac{\left(1-\alpha_{n} v\right) \beta_{n}}{1+\alpha_{n}(v-\rho \tau)}\left\|S x_{n}-x^{*}\right\|^{2} \\
& +\frac{\left(1-\alpha_{n} v\right)\left(1-\beta_{n}\right)}{1+\alpha_{n}(v-\rho \tau)}\left\{\left\|x_{n}-x^{*}\right\|^{2}-\left\|u_{n}-z_{n}\right\|^{2}\right. \\
& \left.+2 \lambda_{n}\left\|u_{n}-z_{n}\right\|\left\|\sum_{i=1}^{N} b_{i} A_{i} u_{n}-\sum_{i=1}^{N} b_{i} A_{i} x^{*}\right\|\right\} \\
\leq & \frac{\alpha_{n} \rho \tau}{1+\alpha_{n}(v-\rho \tau)}\left\|x_{n}-x^{*}\right\|^{2}+\frac{\left(1-\alpha_{n} v\right) \beta_{n}}{1+\alpha_{n}(v-\rho \tau)}\left\|S x_{n}-x^{*}\right\|^{2} \\
& +\frac{2 \alpha_{n}}{1+\alpha_{n}(v-\rho \tau)}\left\langle\rho U\left(x^{*}\right)-\mu F\left(x^{*}\right), P_{C}\left[V_{n}\right]-x^{*}\right\rangle+\left\|x_{n}-x^{*}\right\|^{2} \\
& +\frac{\left(1-\alpha_{n} v\right)\left(1-\beta_{n}\right)}{1+\alpha_{n}(v-\rho \tau)}\left\{-\left\|u_{n}-z_{n}\right\|^{2}\right. \\
& \left.+2 \lambda_{n}\left\|u_{n}-z_{n}\right\|\left\|\sum_{i=1}^{N} b_{i} A_{i} u_{n}-\sum_{i=1}^{N} b_{i} A_{i} x^{*}\right\|\right\} .
\end{aligned}
$$

Hence,

$$
\begin{aligned}
& \frac{\left(1-\alpha_{n} v\right)\left(1-\beta_{n}\right)}{1+\alpha_{n}(v-\rho \tau)}\left\|u_{n}-z_{n}\right\|^{2} \\
& \leq \frac{\alpha_{n} \rho \tau}{1+\alpha_{n}(v-\rho \tau)}\left\|x_{n}-x^{*}\right\|^{2}+\frac{\left(1-\alpha_{n} \nu\right) \beta_{n}}{1+\alpha_{n}(v-\rho \tau)}\left\|S x_{n}-x^{*}\right\|^{2} \\
& \quad+\frac{2 \alpha_{n}}{1+\alpha_{n}(v-\rho \tau)}\left\langle\rho U\left(x^{*}\right)-\mu F\left(x^{*}\right), P_{C}\left[V_{n}\right]-x^{*}\right\rangle \\
& \quad+\left\|x_{n}-x^{*}\right\|^{2}-\left\|P_{C}\left[V_{n}\right]-x^{*}\right\|^{2} \\
& \quad+\frac{\left(1-\alpha_{n} v\right)\left(1-\beta_{n}\right)}{1+\alpha_{n}(\nu-\rho \tau)}\left\{2 \lambda_{n}\left\|u_{n}-z_{n}\right\|\left\|\sum_{i=1}^{N} b_{i} A_{i} u_{n}-\sum_{i=1}^{N} b_{i} A_{i} x^{*}\right\|\right\}
\end{aligned}
$$




$$
\begin{aligned}
\leq & \frac{\alpha_{n} \rho \tau}{1+\alpha_{n}(\nu-\rho \tau)}\left\|x_{n}-x^{*}\right\|^{2}+\frac{\left(1-\alpha_{n} v\right) \beta_{n}}{1+\alpha_{n}(v-\rho \tau)}\left\|S x_{n}-x^{*}\right\|^{2} \\
& +\frac{2 \alpha_{n}}{1+\alpha_{n}(v-\rho \tau)}\left\langle\rho U\left(x^{*}\right)-\mu F\left(x^{*}\right), P_{C}\left[V_{n}\right]-x^{*}\right\rangle \\
& +\left(\left\|x_{n}-x^{*}\right\|+\left\|P_{C}\left[V_{n}\right]-x^{*}\right\|\right)\left\|P_{C}\left[V_{n}\right]-x_{n}\right\| \\
& +2 \lambda_{n}\left\|u_{n}-z_{n}\right\|\left\|\sum_{i=1}^{N} b_{i} A_{i} u_{n}-\sum_{i=1}^{N} b_{i} A_{i} x^{*}\right\| .
\end{aligned}
$$

Since $\lim _{n \rightarrow \infty}\left\|P_{C}\left[V_{n}\right]-x_{n}\right\|=0, \alpha_{n} \rightarrow 0, \beta_{n} \rightarrow 0$, and $\lim _{n \rightarrow \infty} \lambda_{n}=0$, we obtain

$$
\lim _{n \rightarrow \infty}\left\|u_{n}-z_{n}\right\|=0
$$

It follows from (3.19) and (3.20) that

$$
\lim _{n \rightarrow \infty}\left\|x_{n}-z_{n}\right\|=0
$$

Since $T\left(x_{n}\right) \in C$, we have

$$
\begin{aligned}
\left\|x_{n}-T\left(x_{n}\right)\right\| \leq & \left\|x_{n}-x_{n+1}\right\|+\left\|x_{n+1}-T\left(x_{n}\right)\right\| \\
= & \left\|x_{n}-x_{n+1}\right\|+\left\|\gamma_{n}\left(x_{n}-T\left(x_{n}\right)\right)+\left(1-\gamma_{n}\right)\left(P_{C}\left[V_{n}\right]-T\left(x_{n}\right)\right)\right\| \\
\leq & \left\|x_{n}-x_{n+1}\right\|+\gamma_{n}\left\|x_{n}-T\left(x_{n}\right)\right\| \\
& +\left(1-\gamma_{n}\right)\left\|\alpha_{n}\left(\rho U\left(x_{n}\right)-\mu F\left(T\left(y_{n}\right)\right)\right)+T\left(y_{n}\right)-T\left(x_{n}\right)\right\| \\
\leq & \left\|x_{n}-x_{n+1}\right\|+\gamma_{n}\left\|x_{n}-T\left(x_{n}\right)\right\| \\
& +\alpha_{n}\left(1-\gamma_{n}\right)\left\|\rho U\left(x_{n}\right)-\mu F\left(T\left(y_{n}\right)\right)\right\|+\left(1-\gamma_{n}\right)\left\|y_{n}-x_{n}\right\| \\
= & \left\|x_{n}-x_{n+1}\right\|+\gamma_{n}\left\|x_{n}-T\left(x_{n}\right)\right\|+\alpha_{n}\left(1-\gamma_{n}\right)\left\|\rho U\left(x_{n}\right)-\mu F\left(T\left(y_{n}\right)\right)\right\| \\
& +\left(1-\gamma_{n}\right)\left\|\beta_{n} S x_{n}+\left(1-\beta_{n}\right) z_{n}-x_{n}\right\| \\
\leq & \left\|x_{n}-x_{n+1}\right\|+\gamma_{n}\left\|x_{n}-T\left(x_{n}\right)\right\|+\alpha_{n}\left(1-\gamma_{n}\right)\left\|\rho U\left(x_{n}\right)-\mu F\left(T\left(y_{n}\right)\right)\right\| \\
& +\beta_{n}\left(1-\gamma_{n}\right)\left\|S x_{n}-x_{n}\right\|+\left(1-\beta_{n}\right)\left(1-\gamma_{n}\right)\left\|z_{n}-x_{n}\right\|,
\end{aligned}
$$

which implies that

$$
\begin{aligned}
\left\|x_{n}-T\left(x_{n}\right)\right\| \leq & \frac{1}{1-\gamma_{n}}\left\|x_{n}-x_{n+1}\right\|+\alpha_{n}\left\|\rho U\left(x_{n}\right)-\mu F\left(T\left(y_{n}\right)\right)\right\| \\
& +\beta_{n}\left\|S x_{n}-x_{n}\right\|+\left(1-\beta_{n}\right)\left\|z_{n}-x_{n}\right\| .
\end{aligned}
$$

Since $\lim _{n \rightarrow \infty}\left\|x_{n+1}-x_{n}\right\|=0, \alpha_{n} \rightarrow 0, \beta_{n} \rightarrow 0$, and $\left\|\rho U\left(x_{n}\right)-\mu F\left(T\left(y_{n}\right)\right)\right\|$ and $\left\|S x_{n}-x_{n}\right\|$ are bounded, and $\lim _{n \rightarrow \infty}\left\|z_{n}-x_{n}\right\|=0$, we obtain

$$
\lim _{n \rightarrow \infty}\left\|x_{n}-T\left(x_{n}\right)\right\|=0
$$

Since $\left\{x_{n}\right\}$ is bounded, without loss of generality we can assume that $x_{n} \rightarrow x^{*} \in C$. It follows from Lemma 2.4 that $x^{*} \in F(T)$. Therefore $w_{w}\left(x_{n}\right) \subset F(T)$. 
Theorem 3.1 The sequence $\left\{x_{n}\right\}$ generated by Algorithm 3.1 converges strongly to $z$, which is the unique solution of the variational inequality

$$
\langle\rho U(z)-\mu F(z), x-z\rangle \leq 0, \quad \forall x \in F(T) \cap \bigcap_{i=1}^{N} \operatorname{EP}\left(F_{i}\right) \bigcap_{i=1}^{N} \operatorname{VI}\left(C, A_{i}\right) .
$$

Proof Since $\left\{x_{n}\right\}$ is bounded $x_{n} \rightarrow w$ and from Lemma 3.2, we have $w \in F(T)$. Next, we show that $w \in \bigcap_{i=1}^{N} \operatorname{EP}\left(F_{i}\right)$. Since $u_{n}=T_{r_{n}}\left(x_{n}\right)$, we have

$$
\sum_{i=1}^{N} a_{i} F_{i}\left(u_{n}, y\right)+\frac{1}{r_{n}}\left\langle y-u_{n}, u_{n}-x_{n}\right\rangle \geq 0, \quad \forall y \in C .
$$

It follows from the monotonicity of $\sum_{i=1}^{N} a_{i} F_{i}$ that

$$
\frac{1}{r_{n}}\left\langle y-u_{n}, u_{n}-x_{n}\right\rangle \geq \sum_{i=1}^{N} a_{i} F_{i}\left(y, u_{n}\right), \quad \forall y \in C
$$

and

$$
\left\langle y-u_{n_{k}}, \frac{u_{n_{k}}-x_{n_{k}}}{r_{n_{k}}}\right\rangle \geq \sum_{i=1}^{N} a_{i} F_{i}\left(y, u_{n_{k}}\right), \quad \forall y \in C .
$$

Since $\lim _{n \rightarrow \infty}\left\|u_{n}-x_{n}\right\|=0$, and $x_{n} \rightarrow w$, it is easy to observe that $u_{n_{k}} \rightarrow w$. For any $0<$ $t \leq 1$ and $y \in C$, let $y_{t}=t y+(1-t) w$, and let us have $y_{t} \in C$. Then from (3.23), we obtain

$$
0 \geq-\left\langle y_{t}-u_{n_{k}}, \frac{u_{n_{k}}-x_{n_{k}}}{r_{n_{k}}}\right\rangle+\sum_{i=1}^{N} a_{i} F_{i}\left(y_{t}, u_{n_{k}}\right)
$$

Since $u_{n_{k}} \rightarrow w$, it follows from (3.24) that

$$
0 \geq \sum_{i=1}^{N} a_{i} F_{i}\left(y_{t}, w\right)
$$

Since $\sum_{i=1}^{N} a_{i} F_{i}$ satisfies $\left(\mathrm{A}_{1}\right)-\left(\mathrm{A}_{4}\right)$, it follows from (3.25) that

$$
\begin{aligned}
0 & =\sum_{i=1}^{N} a_{i} F_{i}\left(y_{t}, y_{t}\right) \leq t \sum_{i=1}^{N} a_{i} F_{i}\left(y_{t}, y\right)+(1-t) \sum_{i=1}^{N} a_{i} F_{i}\left(y_{t}, w\right) \\
& \leq t \sum_{i=1}^{N} a_{i} F_{i}\left(y_{t}, y\right),
\end{aligned}
$$

which implies that $\sum_{i=1}^{N} a_{i} F_{i}\left(y_{t}, y\right) \geq 0$. Letting $t \rightarrow 0_{+}$, we have

$$
\sum_{i=1}^{N} a_{i} F_{i}(w, y) \geq 0, \quad \forall y \in C,
$$

therefore, $w \in \operatorname{EP}\left(\sum_{i=1}^{N} a_{i} F_{i}\right)=\bigcap_{i=1}^{N} \operatorname{EP}\left(F_{i}\right)$. 
Furthermore, we show that $w \in \bigcap_{i=1}^{N} \operatorname{VI}\left(C, A_{i}\right)$. Let

$$
T v= \begin{cases}\sum_{i=1}^{N} b_{i} A_{i} v+N_{C} v, & \forall v \in C, \\ \emptyset, & \text { otherwise }\end{cases}
$$

where $N_{C} v:=\{w \in H:\langle w, v-u\rangle \geq 0, \forall u \in C\}$ is the normal cone to $C$ at $v \in C$. Then $T$ is maximal monotone and $0 \in T v$ if and only if $v \in \mathrm{VI}\left(C, \sum_{i=1}^{N} b_{i} A_{i}\right)$ (see [33]). Let $G(T)$ denote the graph of $T$, and let $(v, u) \in G(T)$; since $u-\sum_{i=1}^{N} b_{i} A_{i} v \in N_{C} v$ and $z_{n} \in C$, we have

$$
\left\langle v-z_{n}, u-\sum_{i=1}^{N} b_{i} A_{i} v\right\rangle \geq 0
$$

It follows from $z_{n}=P_{C}\left[u_{n}-\lambda_{n} \sum_{i=1}^{N} b_{i} A_{i} u_{n}\right]$ and $v \in C$ that

$$
\left\langle v-z_{n}, z_{n}-\left(u_{n}-\lambda_{n} \sum_{i=1}^{N} b_{i} A_{i} u_{n}\right)\right\rangle \geq 0
$$

and

$$
\left\langle v-z_{n}, \frac{z_{n}-u_{n}}{\lambda_{n}}+\sum_{i=1}^{N} b_{i} A_{i} u_{n}\right\rangle \geq 0 .
$$

Therefore, from (3.27) and strongly positivity of $\sum_{i=1}^{N} b_{i} A_{i}$, we have

$$
\begin{aligned}
\left\langle v-z_{n_{k}}, u\right\rangle \geq & \left\langle v-z_{n_{k}}, \sum_{i=1}^{N} b_{i} A_{i} v\right\rangle \\
\geq & \left\langle v-z_{n_{k}}, \sum_{i=1}^{N} b_{i} A_{i} v\right\rangle-\left\langle v-z_{n_{k}}, \frac{z_{n_{k}}-u_{n_{k}}}{\lambda_{n_{k}}}+\sum_{i=1}^{N} b_{i} A_{i} u_{n_{k}}\right\rangle \\
= & \left\langle v-z_{n_{k}}, \sum_{i=1}^{N} b_{i} A_{i} v-\sum_{i=1}^{N} b_{i} A_{i} z_{n_{k}}\right\rangle+\left\langle v-z_{n_{k}}, \sum_{i=1}^{N} b_{i} A_{i} z_{n_{k}}-\sum_{i=1}^{N} b_{i} A_{i} u_{n_{k}}\right\rangle \\
& -\left\langle v-z_{n_{k}}, \frac{z_{n_{k}}-u_{n_{k}}}{\lambda_{n_{k}}}\right\rangle \\
= & \left\langle v-z_{n_{k}}, \sum_{i=1}^{N} b_{i} A_{i}\left(v-z_{n_{k}}\right)\right\rangle+\left\langle v-z_{n_{k}}, \sum_{i=1}^{N} b_{i} A_{i} z_{n_{k}}-\sum_{i=1}^{N} b_{i} A_{i} u_{n_{k}}\right\rangle \\
& -\left\langle v-z_{n_{k}}, \frac{z_{n_{k}}-u_{n_{k}}}{\lambda_{n_{k}}}\right\rangle \\
\geq & \left\langle v-z_{n_{k}}, \sum_{i=1}^{N} b_{i} A_{i} z_{n_{k}}-\sum_{i=1}^{N} b_{i} A_{i} u_{n_{k}}\right\rangle-\left\langle v-z_{n_{k}}, \frac{z_{n_{k}}-u_{n_{k}}}{\lambda_{n_{k}}}\right\rangle .
\end{aligned}
$$

Since $\lim _{n \rightarrow \infty}\left\|u_{n}-z_{n}\right\|=0$ and $u_{n_{k}} \rightarrow w$, it is easy to observe that $z_{n_{k}} \rightarrow w$. Hence, we obtain $\langle v-w, u\rangle \geq 0$. Since $T$ is maximal monotone, we have $w \in T^{-1} 0$, and hence, $w \in$ 
$\mathrm{VI}\left(C, \sum_{i=1}^{N} b_{i} A_{i}\right)=\bigcap_{i=1}^{N} \operatorname{VI}\left(C, A_{i}\right)$. Thus we have

$$
w \in F(T) \cap \bigcap_{i=1}^{N} \operatorname{EP}\left(F_{i}\right) \cap \bigcap_{i=1}^{N} \operatorname{VI}\left(C, A_{i}\right) .
$$

Observe that the constants satisfy $0 \leq \rho \tau<v$ and

$$
\begin{aligned}
k \geq \eta & \Longleftrightarrow k^{2} \geq \eta^{2} \\
& \Longleftrightarrow 1-2 \mu \eta+\mu^{2} k^{2} \geq 1-2 \mu \eta+\mu^{2} \eta^{2} \\
& \Longleftrightarrow \sqrt{1-\mu\left(2 \eta-\mu k^{2}\right)} \geq 1-\mu \eta \\
& \Longleftrightarrow \mu \eta \geq 1-\sqrt{1-\mu\left(2 \eta-\mu k^{2}\right)} \\
& \Longleftrightarrow \mu \eta \geq v,
\end{aligned}
$$

therefore, from Lemma 2.5, the operator $\mu F-\rho U$ is $\mu \eta-\rho \tau$ strongly monotone, and we get the uniqueness of the solution of the variational inequality (3.22) and denote it by $z \in F(T) \cap \bigcap_{i=1}^{N} \operatorname{EP}\left(F_{i}\right) \bigcap_{i=1}^{N} \operatorname{VI}\left(C, A_{i}\right)$.

Next, we claim that $\limsup _{n \rightarrow \infty}\left\langle\rho U(z)-\mu F(z), x_{n}-z\right\rangle \leq 0$. Since $\left\{x_{n}\right\}$ is bounded, there exists a subsequence $\left\{x_{n_{k}}\right\}$ of $\left\{x_{n}\right\}$ such that

$$
\begin{aligned}
\limsup _{n \rightarrow \infty}\left\langle\rho U(z)-\mu F(z), x_{n}-z\right\rangle & =\limsup _{k \rightarrow \infty}\left\langle\rho U(z)-\mu F(z), x_{n_{k}}-z\right\rangle \\
& =\langle\rho U(z)-\mu F(z), w-z\rangle \leq 0 .
\end{aligned}
$$

By (3.17), we deduce

$$
\begin{aligned}
& \limsup _{n \rightarrow \infty}\left\langle\rho U(z)-\mu F(z), P_{C}\left[V_{n}\right]-z\right\rangle \\
& \quad \leq \limsup _{n \rightarrow \infty}\left\langle\rho U(z)-\mu F(z), P_{C}\left[V_{n}\right]-x_{n}\right\rangle+\limsup _{n \rightarrow \infty}\left\langle\rho U(z)-\mu F(z), x_{n}-z\right\rangle \\
& \quad \leq \limsup _{n \rightarrow \infty}\left\langle\rho U(z)-\mu F(z), x_{n}-z\right\rangle \leq 0 .
\end{aligned}
$$

Next, we show that $x_{n} \rightarrow z$. Note that

$$
\begin{aligned}
\left\|P_{C}\left[V_{n}\right]-z\right\|^{2}= & \left\langle P_{C}\left[V_{n}\right]-z, P_{C}\left[V_{n}\right]-z\right\rangle \\
= & \left\langle P_{C}\left[V_{n}\right]-V_{n}, P_{C}\left[V_{n}\right]-z\right\rangle+\left\langle V_{n}-z, P_{C}\left[V_{n}\right]-z\right\rangle \\
\leq & \left\langle\alpha_{n}\left(\rho U\left(x_{n}\right)-\mu F(z)\right)+\left(I-\alpha_{n} \mu F\right)\left(T\left(y_{n}\right)\right)\right. \\
& \left.-\left(I-\alpha_{n} \mu F\right)(T(z)), P_{C}\left[V_{n}\right]-z\right\rangle \\
= & \left\langle\alpha_{n} \rho\left(U\left(x_{n}\right)-U(z)\right), P_{C}\left[V_{n}\right]-z\right\rangle+\alpha_{n}\left\langle\rho U(z)-\mu F(z), P_{C}\left[V_{n}\right]-z\right\rangle \\
& +\left\langle\left(I-\alpha_{n} \mu F\right)\left(T\left(y_{n}\right)\right)-\left(I-\alpha_{n} \mu F\right)(T(z)), P_{C}\left[V_{n}\right]-z\right\rangle \\
\leq & \alpha_{n} \rho \tau\left\|x_{n}-z\right\|\left\|P_{C}\left[V_{n}\right]-z\right\|+\alpha_{n}\left\langle\rho U(z)-\mu F(z), P_{C}\left[V_{n}\right]-z\right\rangle \\
& +\left(1-\alpha_{n} v\right)\left\|y_{n}-z\right\|\left\|P_{C}\left[V_{n}\right]-z\right\|
\end{aligned}
$$




$$
\begin{aligned}
\leq & \alpha_{n} \rho \tau\left\|x_{n}-z\right\|\left\|P_{C}\left[V_{n}\right]-z\right\|+\alpha_{n}\left\langle\rho U(z)-\mu F(z), P_{C}\left[V_{n}\right]-z\right\rangle \\
& +\left(1-\alpha_{n} \nu\right)\left\{\beta_{n}\left\|S x_{n}-S z\right\|+\beta_{n}\|S z-z\|\right. \\
& \left.+\left(1-\beta_{n}\right)\left\|z_{n}-z\right\|\right\}\left\|P_{C}\left[V_{n}\right]-z\right\| \\
\leq & \alpha_{n} \rho \tau\left\|x_{n}-z\right\|\left\|P_{C}\left[V_{n}\right]-z\right\|+\alpha_{n}\left\langle\rho U(z)-\mu F(z), P_{C}\left[V_{n}\right]-z\right\rangle \\
& +\left(1-\alpha_{n} v\right)\left\{\beta_{n}\left\|x_{n}-z\right\|+\beta_{n}\|S z-z\|\right. \\
& \left.+\left(1-\beta_{n}\right)\left\|x_{n}-z\right\|\right\}\left\|P_{C}\left[V_{n}\right]-z\right\| \\
= & \left(1-\alpha_{n}(v-\rho \tau)\right)\left\|x_{n}-z\right\|\left\|P_{C}\left[V_{n}\right]-z\right\| \\
& +\alpha_{n}\left\langle\rho U(z)-\mu F(z), P_{C}\left[V_{n}\right]-z\right\rangle \\
& +\left(1-\alpha_{n} v\right) \beta_{n}\|S z-z\|\left\|P_{C}\left[V_{n}\right]-z\right\| \\
\leq & \frac{1-\alpha_{n}(v-\rho \tau)}{2}\left(\left\|x_{n}-z\right\|^{2}+\left\|P_{C}\left[V_{n}\right]-z\right\|^{2}\right) \\
& +\alpha_{n}\left\langle\rho U(z)-\mu F(z), P_{C}\left[V_{n}\right]-z\right\rangle \\
& +\left(1-\alpha_{n} v\right) \beta_{n}\|S z-z\|\left\|P_{C}\left[V_{n}\right]-z\right\|,
\end{aligned}
$$

which implies that

$$
\begin{aligned}
\left\|P_{C}\left[V_{n}\right]-z\right\|^{2} \leq & \frac{1-\alpha_{n}(v-\rho \tau)}{1+\alpha_{n}(v-\rho \tau)}\left\|x_{n}-z\right\|^{2} \\
& +\frac{2 \alpha_{n}}{1+\alpha_{n}(v-\rho \tau)}\left\langle\rho U(z)-\mu F(z), P_{C}\left[V_{n}\right]-z\right\rangle \\
& +\frac{2\left(1-\alpha_{n} v\right) \beta_{n}}{1+\alpha_{n}(v-\rho \tau)}\|S z-z\|\left\|P_{C}\left[V_{n}\right]-z\right\| \\
\leq & \left(1-\alpha_{n}(v-\rho \tau)\right)\left\|x_{n}-z\right\|^{2} \\
& +\frac{2 \alpha_{n}(v-\rho \tau)}{1+\alpha_{n}(v-\rho \tau)}\left\{\frac{1}{v-\rho \tau}\left\langle\rho U(z)-\mu F(z), P_{C}\left[V_{n}\right]-z\right\rangle\right. \\
& \left.+\frac{\left(1-\alpha_{n} v\right) \beta_{n}}{\alpha_{n}(v-\rho \tau)}\|S z-z\|\left\|P_{C}\left[V_{n}\right]-z\right\|\right\} .
\end{aligned}
$$

From (3.1) and the inequality above, we get

$$
\begin{aligned}
\left\|x_{n+1}-z\right\|^{2} \leq & \gamma_{n}\left\|x_{n}-z\right\|^{2}+\left(1-\gamma_{n}\right)\left\|P_{C}\left(V_{n}\right)-z\right\|^{2} \\
\leq & \gamma_{n}\left\|x_{n}-z\right\|^{2}+\left(1-\alpha_{n}(v-\rho \tau)\right)\left(1-\gamma_{n}\right)\left\|x_{n}-z\right\|^{2} \\
& +\frac{2 \alpha_{n}\left(1-\gamma_{n}\right)(v-\rho \tau)}{1+\alpha_{n}(v-\rho \tau)}\left\{\frac{1}{v-\rho \tau}\left\{\rho U(z)-\mu F(z), P_{C}\left[V_{n}\right]-z\right\rangle\right. \\
& \left.+\frac{\left(1-\alpha_{n} v\right) \beta_{n}}{\alpha_{n}(v-\rho \tau)}\|S z-z\|\left\|P_{C}\left[V_{n}\right]-z\right\|\right\} \\
= & \left(1-\alpha_{n}(v-\rho \tau)\left(1-\gamma_{n}\right)\right)\left\|x_{n}-z\right\|^{2} \\
& +\frac{2 \alpha_{n}\left(1-\gamma_{n}\right)(v-\rho \tau)}{1+\alpha_{n}(v-\rho \tau)}\left\{\frac{1}{v-\rho \tau}\left\langle\rho U(z)-\mu F(z), P_{C}\left[V_{n}\right]-z\right\rangle\right. \\
& \left.+\frac{\left(1-\alpha_{n} v\right) \beta_{n}}{\alpha_{n}(v-\rho \tau)}\|S z-z\|\left\|P_{C}\left[V_{n}\right]-z\right\|\right\} .
\end{aligned}
$$


Let

$$
v_{n}=\alpha_{n}\left(1-\gamma_{n}\right)(v-\rho \tau)
$$

and

$$
\begin{aligned}
\delta_{n}= & \frac{2 \alpha_{n}\left(1-\gamma_{n}\right)(\nu-\rho \tau)}{1+\alpha_{n}(\nu-\rho \tau)}\left\{\frac{1}{v-\rho \tau}\left\langle\rho U(z)-\mu F(z), P_{C}\left[V_{n}\right]-z\right\rangle\right. \\
& \left.+\frac{\left(1-\alpha_{n} v\right) \beta_{n}}{\alpha_{n}(v-\rho \tau)}\|S z-z\|\left\|P_{C}\left[V_{n}\right]-z\right\|\right\} .
\end{aligned}
$$

We have

$$
\sum_{n=1}^{\infty} \alpha_{n}=\infty
$$

and

$$
\limsup _{n \rightarrow \infty}\left\{\frac{1}{v-\rho \tau}\left\langle\rho U(z)-\mu F(z), P_{C}\left[V_{n}\right]-z\right\rangle+\frac{\left(1-\alpha_{n} v\right) \beta_{n}}{\alpha_{n}(v-\rho \tau)}\|S z-z\|\left\|P_{C}\left[V_{n}\right]-z\right\|\right\} \leq 0 .
$$

It follows that

$$
\sum_{n=1}^{\infty} v_{n}=\infty \text { and } \quad \limsup _{n \rightarrow \infty} \frac{\delta_{n}}{v_{n}} \leq 0
$$

Thus all the conditions of Lemma 2.7 are satisfied. Hence we deduce that $x_{n} \rightarrow z$. This completes the proof.

\section{Applications}

To verify the theoretical assertions, we consider the following examples.

Example 4.1 Let $\alpha_{n}=\frac{1}{3 n}, \gamma_{n}=\frac{1}{2 n}, \beta_{n}=\frac{1}{n^{3}}, \lambda_{n}=\frac{1}{8(n+1)}$, and $r_{n}=\frac{n}{n+1}$.

We have

$$
\lim _{n \rightarrow \infty} \alpha_{n}=\frac{1}{3} \lim _{n \rightarrow \infty} \frac{1}{n}=0
$$

and

$$
\sum_{n=1}^{\infty} \alpha_{n}=\frac{1}{3} \sum_{n=1}^{\infty} \frac{1}{n}=\infty
$$

The sequence $\left\{\alpha_{n}\right\}$ satisfies condition (b),

$$
\lim _{n \rightarrow \infty} \frac{\beta_{n}}{\alpha_{n}}=\lim _{n \rightarrow \infty} \frac{3}{n^{2}}=0 .
$$

Condition (c) is satisfied. We compute

$$
\alpha_{n-1}-\alpha_{n}=\frac{1}{3}\left(\frac{1}{n-1}-\frac{1}{n}\right)=\frac{1}{3 n(n-1)} .
$$


It is easy to show $\sum_{n=1}^{\infty}\left|\alpha_{n-1}-\alpha_{n}\right|<\infty$. Similarly, we can show $\sum_{n=1}^{\infty}\left|\gamma_{n-1}-\gamma_{n}\right|<\infty$ and $\sum_{n=1}^{\infty}\left|\beta_{n-1}-\beta_{n}\right|<\infty$. The sequences $\left\{\alpha_{n}\right\},\left\{\gamma_{n}\right\}$ and $\left\{\beta_{n}\right\}$ satisfy condition (e). We have

$$
\liminf _{n \rightarrow \infty} r_{n}=\liminf _{n \rightarrow \infty} \frac{n}{n+1}=1
$$

and

$$
\begin{aligned}
\sum_{n=1}^{\infty}\left|r_{n-1}-r_{n}\right| & =\sum_{n=1}^{\infty}\left|\frac{n-1}{n}-\frac{n}{n+1}\right| \\
& =\sum_{n=1}^{\infty} \frac{1}{n(n+1)} \\
& \leq \sum_{n=1}^{\infty} \frac{1}{n^{2}} \\
& <\infty .
\end{aligned}
$$

Then the sequence $\left\{r_{n}\right\}$ satisfies condition (f),

$$
\begin{aligned}
\sum_{n=1}^{\infty}\left|\lambda_{n-1}-\lambda_{n}\right| & =\sum_{n=1}^{\infty}\left|\frac{1}{8 n}-\frac{1}{8(n+1)}\right| \\
& =\frac{1}{8} \sum_{n=1}^{\infty}\left|\frac{1}{n}-\frac{1}{n+1}\right| \\
& \leq \frac{1}{8}
\end{aligned}
$$

Then the sequence $\left\{\lambda_{n}\right\}$ satisfies condition $(\mathrm{g})$.

Let $\mathbb{R}$ be the set of real numbers, and let the mapping $T: \mathbb{R} \rightarrow \mathbb{R}$ be defined by

$$
T(x)=\frac{x}{2}, \quad \forall x \in \mathbb{R}
$$

let the mapping $F: \mathbb{R} \rightarrow \mathbb{R}$ be defined by

$$
F(x)=\frac{2 x+3}{7}, \quad \forall x \in \mathbb{R}
$$

let the mapping $S: \mathbb{R} \rightarrow \mathbb{R}$ be defined by

$$
S(x)=\frac{x}{3}, \quad \forall x \in \mathbb{R}
$$

let the mapping $U: \mathbb{R} \rightarrow \mathbb{R}$ be defined by

$$
U(x)=\frac{x}{14}, \quad \forall x \in \mathbb{R}
$$

and, for $i=1,2, \ldots, N$, let the mapping $A_{i}: \mathbb{R} \rightarrow \mathbb{R}$ be defined by

$$
A_{i} x=\frac{i x}{2}, \quad \forall x \in \mathbb{R}
$$


and $b_{i}=\frac{7}{8^{i}}+\frac{1}{N 8^{N}}$, and let the mapping $F_{i}: \mathbb{R} \times \mathbb{R} \rightarrow \mathbb{R}$ be defined by

$$
F_{i}(x, y)=i\left(-3 x^{2}+x y+2 y^{2}\right), \quad \forall(x, y) \in \mathbb{R} \times \mathbb{R}
$$

and $a_{i}=\frac{2}{3^{i}}+\frac{1}{N 3^{N}}$.

It is easy to show that $T$ and $S$ are nonexpansive mappings, $F$ is a 1-Lipschitzian mapping and $\frac{1}{7}$-strongly monotone, $U$ is a $\frac{1}{7}$-Lipschitzian, $A_{i}$ is a strongly positive linear bounded operator, and the $F_{i}$ satisfy $\left(\mathrm{A}_{1}\right)-\left(\mathrm{A}_{4}\right)$. It is clear that

$$
F(T) \cap \bigcap_{i=1}^{N} \operatorname{EP}\left(F_{i}\right) \cap \bigcap_{i=1}^{N} \operatorname{VI}\left(C, A_{i}\right)=\{0\} .
$$

By the definition of $F_{i}$, we have

$$
\begin{aligned}
0 & \leq \sum_{i=1}^{N} a_{i} F_{i}\left(u_{n}, y\right)+\frac{1}{r_{n}}\left\langle y-u_{n}, u_{n}-x_{n}\right\rangle \\
& =\sigma\left(-3 u_{n}^{2}+u_{n} y+2 y^{2}\right)+\frac{1}{r_{n}}\left(y-u_{n}\right)\left(u_{n}-x_{n}\right),
\end{aligned}
$$

where $\sigma=\sum_{i=1}^{N}\left(\frac{2}{3^{i}}+\frac{1}{N 3^{N}}\right) i$. Then

$$
\begin{aligned}
0 & \leq \sigma r_{n}\left(-3 u_{n}^{2}+u_{n} y+2 y^{2}\right)+\left(y u_{n}-y x_{n}-u_{n}^{2}+u_{n} x_{n}\right) \\
& =2 \sigma r_{n} y^{2}+\left(\sigma r_{n} u_{n}+u_{n}-x_{n}\right) y-3 \sigma r_{n} u_{n}^{2}-u_{n}^{2}+u_{n} x_{n} .
\end{aligned}
$$

Let $B(y)=2 \sigma r_{n} y^{2}+\left(\sigma r_{n} u_{n}+u_{n}-x_{n}\right) y-3 \sigma r_{n} u_{n}^{2}-u_{n}^{2}+u_{n} x_{n} . B(y)$ is a quadratic function of $y$ with coefficient $a=2 \sigma r_{n}, b=\sigma r_{n} u_{n}+u_{n}-x_{n}, c=-3 \sigma r_{n} u_{n}^{2}-u_{n}^{2}+u_{n} x_{n}$. We determine the discriminant $\Delta$ of $B$ as follows:

$$
\begin{aligned}
\Delta & =b^{2}-4 a c \\
& =\left(\sigma r_{n} u_{n}+u_{n}-x_{n}\right)^{2}-8 \sigma r_{n}\left(-3 \sigma r_{n} u_{n}^{2}-u_{n}^{2}+u_{n} x_{n}\right) \\
& =u_{n}^{2}+10 \sigma r_{n} u_{n}^{2}+25 \sigma^{2} u_{n}^{2} r_{n}^{2}-2 x_{n} u_{n}-10 \sigma x_{n} u_{n} r_{n}+x_{n}^{2} \\
& =\left(u_{n}+5 \sigma u_{n} r_{n}\right)^{2}-2 x_{n}\left(u_{n}+5 \sigma u_{n} r_{n}\right)+x_{n}^{2} \\
& =\left(u_{n}+5 \sigma u_{n} r_{n}-x_{n}\right)^{2} .
\end{aligned}
$$

We have $B(y) \geq 0, \forall y \in \mathbb{R}$. If it has at most one solution in $\mathbb{R}$, then $\Delta=0$, we obtain

$$
u_{n}=\frac{x_{n}}{1+5 \sigma r_{n}} \text {. }
$$

For every $n \geq 1$, from (4.1), we rewrite (3.1) as follows:

$$
\left\{\begin{array}{l}
z_{n}=\frac{x_{n}}{1+5 \sigma r_{n}}-\sum_{i=1}^{N} b_{i} \frac{i x_{n}}{16(n+1)\left(1+5 \sigma r_{n}\right)} \\
y_{n}=\frac{x_{n}}{3 n^{3}}+\left(1-\frac{1}{n^{3}}\right) z_{n} \\
x_{n+1}=\frac{x_{n}}{2 n}+\left(1-\frac{1}{2 n}\right)\left(\rho \frac{x_{n}}{42 n}+\frac{y_{n}}{2}-\mu \frac{y_{n}+3}{21 n}\right)
\end{array}\right.
$$


Table 1 The values of $\left\{u_{n}\right\},\left\{z_{n}\right\},\left\{y_{n}\right\}$, and $\left\{x_{n}\right\}$ with initial value $x_{1}=40$

\begin{tabular}{|c|c|c|c|c|c|c|c|c|}
\hline & \multicolumn{4}{|c|}{ Algorithm 3.1} & \multicolumn{4}{|c|}{ Algorithm 3.2} \\
\hline & $u_{n}$ & $z_{n}$ & $y_{n}$ & $x_{n}$ & $u_{n}$ & $z_{n}$ & $y_{n}$ & $x_{n}$ \\
\hline$n=1$ & 8.421503 & 7.218431 & 13.333333 & 40.000000 & 11.428571 & 10.000000 & 13.333333 & 40.000000 \\
\hline$n=2$ & 3.885140 & 3.052610 & 3.642264 & 23.309524 & 5.379121 & 4.370536 & 4.795449 & 23.309524 \\
\hline$n=3$ & 1.085370 & 0.775264 & 0.835318 & 7.190160 & 1.604140 & 1.203105 & 1.252615 & 7.619663 \\
\hline$n=4$ & 0.220346 & 0.141651 & 0.147471 & 1.542332 & 0.357438 & 0.245739 & 0.251207 & 1.787190 \\
\hline$n=5$ & 0.034921 & 0.019955 & 0.020470 & 0.253162 & 0.063694 & 0.039809 & 0.040368 & 0.329084 \\
\hline$n=6$ & 0.004160 & 0.002080 & 0.002118 & 0.030902 & 0.008976 & 0.005049 & 0.005099 & 0.047445 \\
\hline$n=7$ & 0.000057 & 0.000025 & 0.000025 & 0.000433 & 0.000591 & 0.000296 & 0.000298 & 0.003179 \\
\hline$n=8$ & -0.000348 & -0.000124 & -0.000126 & -0.002665 & -0.000430 & -0.000188 & -0.000189 & -0.002341 \\
\hline$n=9$ & -0.000338 & -0.000096 & -0.000098 & -0.002617 & -0.000478 & -0.000179 & -0.000180 & -0.002627 \\
\hline$n=10$ & -0.000298 & -0.000064 & -0.000065 & -0.002333 & -0.000428 & -0.000134 & -0.000134 & -0.002373 \\
\hline
\end{tabular}

Table 2 The values of $\left\{u_{n}\right\},\left\{z_{n}\right\},\left\{y_{n}\right\}$ and $\left\{x_{n}\right\}$ with initial value $x_{1}=-40$

\begin{tabular}{|c|c|c|c|c|c|c|c|c|}
\hline & \multicolumn{4}{|c|}{ Algorithm 3.1} & \multicolumn{4}{|c|}{ Algorithm 3.2} \\
\hline & $u_{n}$ & $z_{n}$ & $y_{n}$ & $x_{n}$ & $u_{n}$ & $z_{n}$ & $y_{n}$ & $x_{n}$ \\
\hline$n=1$ & -8.421503 & -7.218431 & -13.333333 & -40.000000 & -11.428571 & -10.000000 & -13.333333 & -40.000000 \\
\hline$n=2$ & -3.888542 & -3.055283 & -3.645453 & -23.329932 & -5.383830 & -4.374362 & -4.799647 & -23.329932 \\
\hline$n=3$ & -1.088631 & -0.777594 & -0.837828 & -7.211768 & -1.608768 & -1.206576 & -1.256229 & -7.641647 \\
\hline$n=4$ & -0.222630 & -0.143120 & -0.149000 & -1.558322 & -0.360740 & -0.248009 & -0.253528 & -1.803701 \\
\hline$n=5$ & -0.036521 & -0.020869 & -0.021408 & -0.264761 & -0.066018 & -0.041261 & -0.041841 & -0.341094 \\
\hline$n=6$ & -0.005362 & -0.002681 & -0.002730 & -0.039833 & -0.010719 & -0.006029 & -0.006089 & -0.056658 \\
\hline$n=7$ & -0.001018 & -0.000436 & -0.000442 & -0.007695 & -0.001979 & -0.000990 & -0.000997 & -0.010638 \\
\hline$n=8$ & -0.000452 & -0.000161 & -0.000163 & -0.003463 & -0.000722 & -0.000316 & -0.000318 & -0.003931 \\
\hline$n=9$ & -0.000346 & -0.000099 & -0.000100 & -0.002685 & -0.000507 & -0.000190 & -0.000191 & -0.002787 \\
\hline$n=10$ & -0.000299 & -0.000064 & -0.000065 & -0.002338 & -0.000430 & -0.000135 & -0.000135 & -0.002387 \\
\hline
\end{tabular}
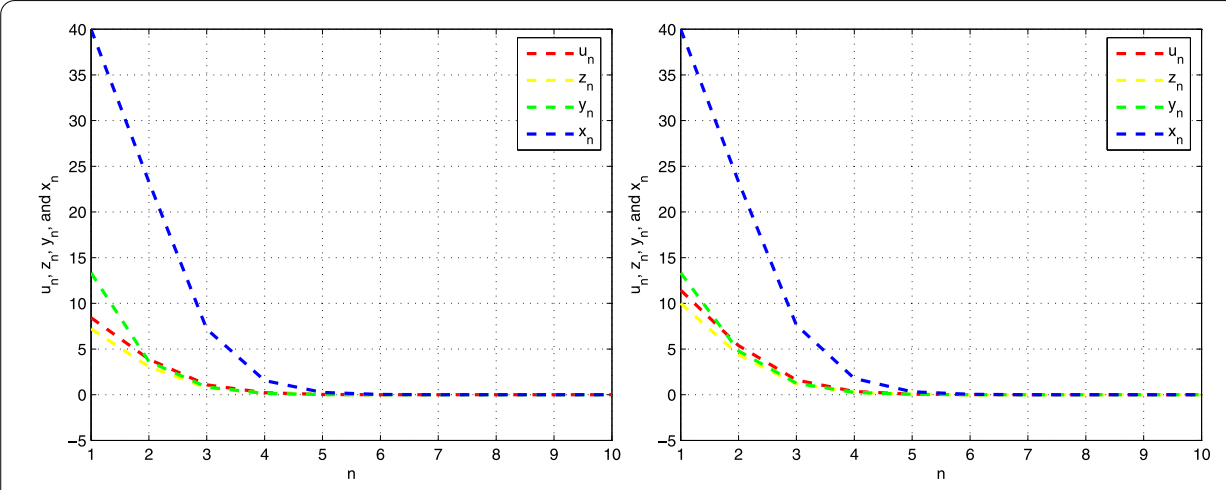

Figure 1 The convergence of $\left\{u_{n}\right\},\left\{z_{n}\right\},\left\{y_{n}\right\}$, and $\left\{x_{n}\right\}$ with initial value $x_{1}=40$ for Algorithm 3.1 and Algorithm 3.2.

In all the tests we take $\rho=\frac{1}{15}$ and $\mu=\frac{1}{7}$. In our example, $\eta=\frac{1}{7}, k=1, \tau=\frac{1}{7}$. It is easy to show that the parameters satisfy $0<\mu<\frac{2 \eta}{k^{2}}, 0 \leq \rho \tau<\nu$, where $v=1-\sqrt{1-\mu\left(2 \eta-\mu k^{2}\right)}$. All codes were written in Matlab, the values of $\left\{u_{n}\right\},\left\{z_{n}\right\},\left\{y_{n}\right\}$, and $\left\{x_{n}\right\}$ with different $n$ are reported in Tables 1 and 2 .

Remark 4.1 Tables 1 and 2, and Figures 1 and 2 show that the sequences $\left\{u_{n}\right\},\left\{z_{n}\right\},\left\{y_{n}\right\}$ and $\left\{x_{n}\right\}$ converge to 0 , where $\{0\}=F(T) \cap \bigcap_{i=1}^{N} \operatorname{EP}\left(F_{i}\right) \cap \bigcap_{i=1}^{N} \operatorname{VI}\left(C, A_{i}\right)$.

Tables 1 and 2 show that the convergence of Algorithm 3.1 is faster than Algorithm 3.2. 


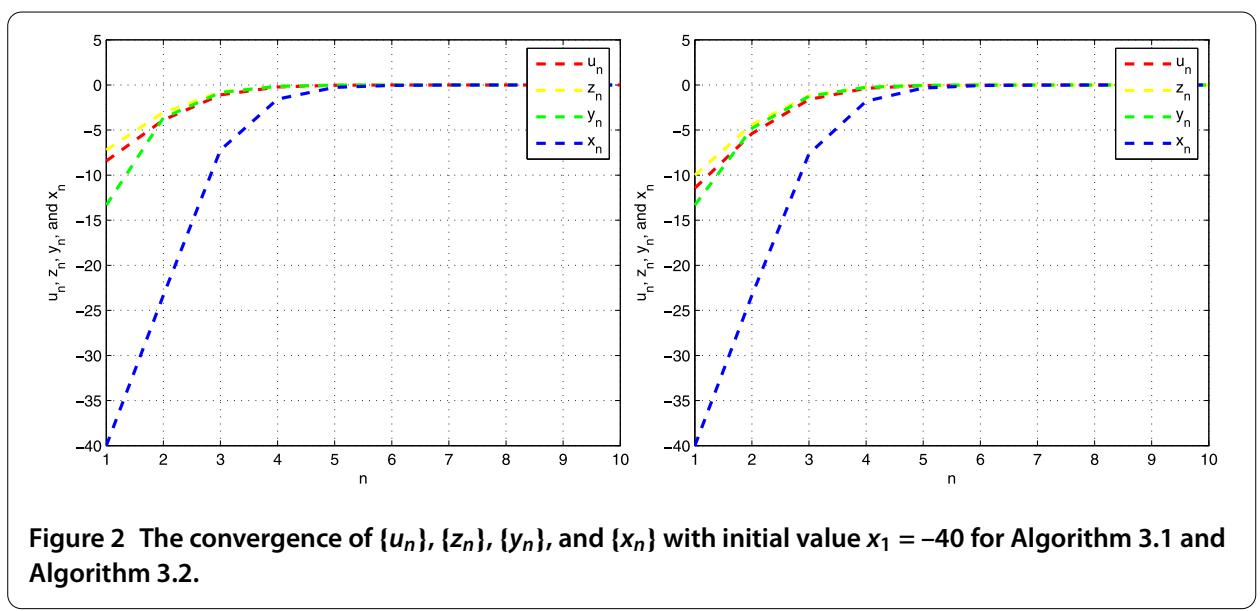

Example 4.2 In this example we take the same mappings and parameters as in Example 4.1 except $T$ and $F_{i}$.

Let $T:[1,70] \rightarrow[1,70]$ be defined by

$$
T(x)=\frac{2 x+5}{7}, \quad \forall x \in[1,70],
$$

and for $i=1,2, \ldots, N$, let the mapping $F_{i}:[1,70] \times[1,70] \rightarrow \mathbb{R}$ be defined by

$$
F_{i}(x, y)=i(y-x)(y+2 x-3), \quad \forall(x, y) \in[1,70] \times[1,70],
$$

and $a_{i}=\frac{4}{5^{i}}+\frac{1}{N 5^{N}}$. It is clear that

$$
F(T) \cap \bigcap_{i=1}^{N} \operatorname{EP}\left(F_{i}\right) \cap \bigcap_{i=1}^{N} \operatorname{VI}\left(C, A_{i}\right)=\{1\} .
$$

By the definition of $F_{i}$, we have

$$
\begin{aligned}
0 & \leq \sum_{i=1}^{N} a_{i} F_{i}\left(u_{n}, y\right)+\frac{1}{r_{n}}\left\langle y-u_{n}, u_{n}-x_{n}\right\rangle \\
& =\omega\left(y-u_{n}\right)\left(y+2 u_{n}-3\right)+\frac{1}{r_{n}}\left(y-u_{n}\right)\left(u_{n}-x_{n}\right),
\end{aligned}
$$

where $\omega=\sum_{i=1}^{N} \frac{4}{5^{i}}+\frac{1}{N 5^{N}} i$. Then

$$
\begin{aligned}
0 & \leq \omega r_{n}\left(y-u_{n}\right)\left(y+2 u_{n}-3\right)+\left(y u_{n}-y x_{n}-u_{n}^{2}+u_{n} x_{n}\right) \\
& =\omega r_{n} y^{2}+\left(\omega r_{n} u_{n}+u_{n}-x_{n}-3 \omega r_{n}\right) y+3 \omega r_{n} u_{n}-u_{n}^{2}-2 \omega r_{n} u_{n}^{2}+u_{n} x_{n} .
\end{aligned}
$$

Let $A(y)=\omega r_{n} y^{2}+\left(\omega r_{n} u_{n}+u_{n}-x_{n}-3 \omega r_{n}\right) y+3 \omega r_{n} u_{n}-u_{n}^{2}-2 \omega r_{n} u_{n}^{2}+u_{n} x_{n} . A(y)$ is a quadratic function of $y$ with coefficient $a=\omega r_{n}, b=\omega r_{n} u_{n}+u_{n}-x_{n}-3 \omega r_{n}, c=3 \omega r_{n} u_{n}-$ $u_{n}^{2}-2 \omega r_{n} u_{n}^{2}+u_{n} x_{n}$. We determine the discriminant $\Delta$ of $A$ as follows:

$$
\begin{aligned}
\Delta & =b^{2}-4 a c \\
& =\left(\omega r_{n} u_{n}+u_{n}-x_{n}-3 \omega r_{n}\right)^{2}-4 \omega r_{n}\left(3 \omega r_{n} u_{n}-u_{n}^{2}-2 \omega r_{n} u_{n}^{2}+u_{n} x_{n}\right)
\end{aligned}
$$




$$
\begin{aligned}
= & 9 \omega^{2} r_{n}^{2}-6 \omega r_{n} u_{n}-18 \omega^{2} r_{n}^{2} u_{n}+u_{n}^{2}+6 \omega r_{n} u_{n}^{2}+9 \omega^{2} r_{n}^{2} u_{n}^{2} \\
& +6 \omega r_{n} x_{n}-2 u_{n} x_{n}-6 \omega r_{n} u_{n} x_{n}+x_{n}^{2} \\
= & \left(u_{n}-3 \omega r_{n}+3 \omega u_{n} r_{n}-x_{n}\right)^{2} .
\end{aligned}
$$

We have $A(y) \geq 0, \forall y \in \mathbb{R}$. If it has at most one solution in $\mathbb{R}$, then $\Delta=0$, we obtain

$$
u_{n}=\frac{x_{n}+3 \omega r_{n}}{1+3 \omega r_{n}}
$$

For every $n \geq 1$, we rewrite (3.1) as follows:

$$
\left\{\begin{array}{l}
z_{n}=P_{[1,70]}\left(u_{n}-\sum_{i=1}^{N} b_{i} \frac{i u_{n}}{16(n+1)}\right) \\
y_{n}=\frac{x_{n}}{3 n^{3}}+\left(1-\frac{1}{n^{3}}\right) z_{n} ; \\
x_{n+1}=\frac{x_{n}}{2 n}+\left(1-\frac{1}{2 n}\right) P_{[1,70]}\left(\rho \frac{x_{n}}{42 n}+\frac{2 y_{n}+5}{7}-\mu \frac{4 y_{n}+31}{147 n}\right) .
\end{array}\right.
$$

\begin{tabular}{|c|c|c|c|c|c|c|c|c|}
\hline & \multicolumn{4}{|c|}{ Algorithm 3.1} & \multicolumn{4}{|c|}{ Algorithm 3.2} \\
\hline & $u_{n}$ & $z_{n}$ & $y_{n}$ & $x_{n}$ & $u_{n}$ & $z_{n}$ & $y_{n}$ & $x_{n}$ \\
\hline$n=1$ & 14.565222 & 12.484476 & 13.333333 & 40.000000 & 16.600000 & 14.525000 & 13.333333 & 40.000000 \\
\hline$n=2$ & 7.061065 & 5.547979 & 5.780054 & 22.213719 & 8.071240 & 6.557882 & 6.663719 & 22.213719 \\
\hline$n=3$ & 2.655057 & 1.896469 & 1.916476 & 7.309903 & 2.998732 & 2.249049 & 2.258293 & 7.495879 \\
\hline$n=4$ & 1.315259 & 1.000000 & 0.996151 & 2.261036 & 1.403685 & 1.000000 & 0.996732 & 2.372530 \\
\hline$n=5$ & 1.038213 & 1.000000 & 0.995087 & 1.157630 & 1.049019 & 1.000000 & 0.995124 & 1.171566 \\
\hline$n=6$ & 1.003740 & 1.000000 & 0.996938 & 1.015763 & 1.004804 & 1.000000 & 0.996940 & 1.017157 \\
\hline$n=7$ & 1.000307 & 1.000000 & 0.998058 & 1.001314 & 1.000394 & 1.000000 & 0.998058 & 1.001430 \\
\hline$n=8$ & 1.000022 & 1.000000 & 0.998698 & 1.000094 & 1.000028 & 1.000000 & 0.998698 & 1.000102 \\
\hline$n=9$ & 1.000001 & 1.000000 & 0.999086 & 1.000006 & 1.000002 & 1.000000 & 0.999086 & 1.000006 \\
\hline$n=10$ & 1.000000 & 1.000000 & 0.999333 & 1.000000 & 1.000000 & 1.000000 & 0.999333 & 1.000000 \\
\hline
\end{tabular}

Remark 4.2 Table 3 and Figure 3 show that the sequences $\left\{u_{n}\right\},\left\{z_{n}\right\},\left\{y_{n}\right\}$, and $\left\{x_{n}\right\}$ converge to 1 , where $\{1\}=F(T) \cap \bigcap_{i=1}^{N} \operatorname{EP}\left(F_{i}\right) \cap \bigcap_{i=1}^{N} \operatorname{VI}\left(C, A_{i}\right)$.

Table 3 shows that the convergence of Algorithm 3.1 is faster than Algorithm 3.2.

Table 3 The values of $\left\{u_{n}\right\},\left\{z_{n}\right\},\left\{y_{n}\right\}$, and $\left\{x_{n}\right\}$ with initial value $x_{1}=40$
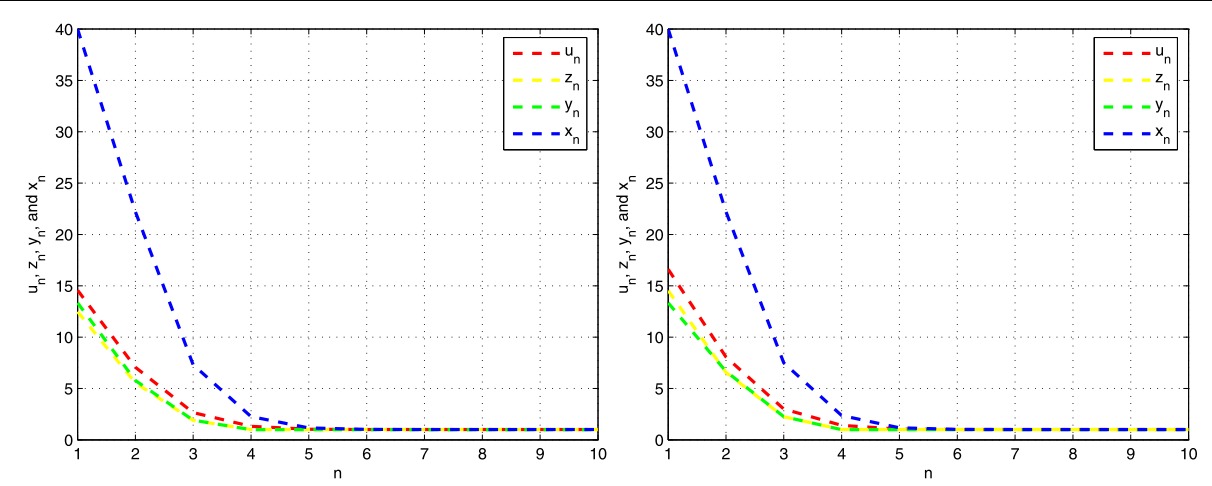

Figure 3 The convergence of $\left\{u_{n}\right\},\left\{z_{n}\right\},\left\{y_{n}\right\}$, and $\left\{x_{n}\right\}$ with initial values $x_{1}=40$ for Algorithm 3.1 and Algorithm 3.2. 


\section{Conclusions}

In this paper, we suggest and analyze an iterative method for finding the approximate element of the common set of solutions of (1.3), (1.4), and (1.8) in real Hilbert space, which can be viewed as a refinement and improvement of some existing methods for solving a variational inequality problem, an equilibrium problem, and a hierarchical fixed point problem. Some existing methods (e.g. $[13,16,18,23,25,27])$ can be viewed as special cases of Algorithm 3.1. Therefore, the new algorithm is expected to be widely applicable. In hierarchical fixed point problem (1.8), if $S=I-(\rho U-\mu F)$, then we can get the variational inequality (3.22).

In (3.22), if $U=0$ then we get the variational inequality

$$
\langle F(z), x-z\rangle \geq 0, \quad \forall x \in F(T) \cap \bigcap_{i=1}^{N} \mathrm{EP}\left(F_{i}\right) \bigcap_{i=1}^{N} \mathrm{VI}\left(C, A_{i}\right),
$$

which just is the variational inequality studied by Suzuki [29] extending the common set of solutions of a combination of variational inequality problems, a combination of equilibria problem, and a hierarchical fixed point problem.

\section{Competing interests}

The author declares that he has no competing interests.

\section{Acknowledgement}

The author would like to thank Professor Omar Halli, Rector, Ibn Zohr University, for providing excellent research facilities.

Received: 11 April 2014 Accepted: 27 June 2014 Published: 23 Jul 2014

\section{References}

1. Blum, E, Oettli, W: From optimization and variational inequalities to equilibrium problems. Math. Stud. 63, 123-145 (1994)

2. Combettes, PL, Hirstoaga, SA: Equilibrium programming using proximal like algorithms. Math. Program. 78, 29-41 (1997)

3. Combettes, PL, Hirstoaga, SA: Equilibrium programming in Hilbert spaces. J. Nonlinear Convex Anal. 6, 117-136 (2005)

4. Ceng, LC, Yao, JC: A hybrid iterative scheme for mixed equilibrium problems and fixed point problems. J. Comput. Appl. Math. 214(1), 186-201 (2008)

5. Takahashi, S, Takahashi, W: Strong convergence theorem for a generalized equilibrium problem and a nonexpansive mapping in a Hilbert space. Nonlinear Anal. 69(3), 1025-1033 (2008)

6. Reich, S, Sabach, S: Three strong convergence theorems regarding iterative methods for solving equilibrium problems in reflexive Banach spaces. In: Optimization Theory and Related Topics. Contemp. Math. vol. 568, pp. 225-240 (2012)

7. Kassay, G, Reich, S, Sabach, S: Iterative methods for solving systems of variational inequalities in reflexive Banach spaces. SIAM J. Optim. 21, 1319-1344 (2011)

8. Chang, SS, Lee, HWJ, Chan, CK: A new method for solving equilibrium problem fixed point problem and variational inequality problem with application to optimization. Nonlinear Anal. 70, 3307-3319 (2009)

9. Qin, X, Shang, M, Su, Y: A general iterative method for equilibrium problem and fixed point problem in Hilbert spaces. Nonlinear Anal. 69(11), 3897-3909 (2008)

10. Latif, A, Ceng, LC, Ansari, QH: Multi-step hybrid viscosity method for systems of variational inequalities defined over sets of solutions of equilibrium problem and fixed point problems. Fixed Point Theory Appl. 2012, 186 (2012)

11. Marino, C, Muglia, L, Yao, JC: Viscosity methods for common solutions of equilibrium and variational inequality problems via multi-step iterative algorithms and common fixed points. Nonlinear Anal. 75(4), 1787-1798 (2012)

12. Suwannaut, S, Kangtunyakarn, A: The combination of the set of solutions of equilibrium problem for convergence theorem of the set of fixed points of strictly pseudo-contractive mappings and variational inequalities problem. Fixed Point Theory Appl. 2013, 291 (2013)

13. Yao, JC, Cho, YJ, Liou, YC: Iterative algorithms for hierarchical fixed points problems and variational inequalities. Math. Comput. Model. 52(9-10), 1697-1705 (2010)

14. Bnouhachem, A: Strong convergence algorithm for split equilibrium problems and hierarchical fixed point problems. Sci. World J. 2014, Article ID 390956 (2014)

15. Bnouhachem, A: Strong convergence algorithm for approximating the common solutions of a variational inequality, a mixed equilibrium problem and a hierarchical fixed-point problem. J. Inequal. Appl. 2014, 154 (2014)

16. Mainge, PE, Moudafi, A: Strong convergence of an iterative method for hierarchical fixed-point problems. Pac. J. Optim. 3(3), 529-538 (2007)

17. Moudafi, A: Krasnoselski-Mann iteration for hierarchical fixed-point problems. Inverse Probl. 23(4), 1635-1640 (2007) 
18. Cianciaruso, F, Marino, G, Muglia, L, Yao, Y: On a two-steps algorithm for hierarchical fixed point problems and variational inequalities. J. Inequal. Appl. 2009, Article ID 208692 (2009)

19. Gu, G, Wang, S, Cho, YJ: Strong convergence algorithms for hierarchical fixed points problems and variational inequalities. J. Appl. Math. 2011, Article ID 164978 (2011)

20. Marino, G, Xu, HK: Explicit hierarchical fixed point approach to variational inequalities. J. Optim. Theory Appl. 149(1), 61-78 (2011)

21. Crombez, G: A hierarchical presentation of operators with fixed points on Hilbert spaces. Numer. Funct. Anal. Optim. 27(3-4), 259-277 (2006)

22. Ceng, LC, Khan, AR, Ansari, QH, Yao, JC: Viscosity approximation methods for strongly positive and monotone operators. Fixed Point Theory 10(1), 35-71 (2009)

23. Ceng, LC, Anasri, QH, Yao, JC: Some iterative methods for finding fixed points and for solving constrained convex minimization problems. Nonlinear Anal. 74(16), 5286-5302 (2011)

24. Wang, $Y, X u, W$ : Strong convergence of a modified iterative algorithm for hierarchical fixed point problems and variational inequalities. Fixed Point Theory Appl. 2013, 121 (2013)

25. Bnouhachem, A: A modified projection method for a common solution of a system of variational inequalities, a split equilibrium problem and a hierarchical fixed-point problem. Fixed Point Theory Appl. 2014, 22 (2014)

26. Ceng, LC, Anasri, QH, Yao, JC: Iterative methods for triple hierarchical variational inequalities in Hilbert spaces. J. Optim. Theory Appl. 151, 489-512 (2011)

27. Tian, M: A general iterative algorithm for nonexpansive mappings in Hilbert spaces. Nonlinear Anal. 73(3), 689-694 (2010)

28. Geobel, K, Kirk, WA: Topics in Metric Fixed Point Theory. Stud. Adv. Math., vol. 28. Cambridge University Press, Cambridge (1990)

29. Suzuki, N: Moudafi's viscosity approximations with Meir-Keeler contractions. J. Math. Anal. Appl. 325(1), 342-352 (2007)

30. Xu, HK: Iterative algorithms for nonlinear operators. J. Lond. Math. Soc. 66(1), 240-256 (2002)

31. Acedo, GL, Xu, HK: Iterative methods for strictly pseudo-contractions in Hilbert spaces. Nonlinear Anal. 67(7), 2258-2271 (2007)

32. Cianciaruso, F, Marino, G, Muglia, L, Yao, Y: A hybrid projection algorithm for finding solutions of mixed equilibrium problem and variational inequality problem. Fixed Point Theory Appl. 2010, Article ID 383740 (2010)

33. Rockafellar, RT: On the maximality of sums nonlinear monotone operators. Trans. Am. Math. Soc. 149, 75-88 (1970)

10.1186/1687-1812-2014-163

Cite this article as: Bnouhachem: A hybrid iterative method for a combination of equilibria problem, a combination of variational inequality problems and a hierarchical fixed point problem. Fixed Point Theory and Applications

2014, 2014:163

\section{Submit your manuscript to a SpringerOpen ${ }^{\ominus}$ journal and benefit from:}

- Convenient online submission

- Rigorous peer review

- Immediate publication on acceptance

Open access: articles freely available online

- High visibility within the field

- Retaining the copyright to your article 